\title{
Gravitational Lensing and Catastrophe Theory
}

\author{
Yuuko KAKIGI, Takashi OKAMURA* and Takeshi FUKUYAMA \\ Department of Physics, \\ Ritsumeikan University, Kusatsu, \\ Shiga, 525 Japan
}

\begin{abstract}
Singularities of caustics appeared in gravitational lensing effect are discussed analytically.

Multipole expansion model of lensing object is mainly studied since it is tractable and universal. Our analyses are confirmed by numerical calculations and applied to multiple quasar system of PG1115+080. Consistencies with elliptical lens models are also discussed.
\end{abstract}

PACS number 95.30.Sf, 98.80.Es

*Present Address : Department of Physics, Tokyo Institute of Technology, Oh-Okayama, Megroku, Tokyo, 152 Japan 


\section{Introduction}

Our starting point in discussing gravitational lensing (hereafter GL) is Fermat's potential [1], [2]. Lens equation is given by the extremum of Fermat's potential and the critical lines are points where the Jacobian from source plane to lens plane vanishes. (See Fig.1)

Fermat's potential is all from which we get information. Assuming thin lens approximation, Fermat's potential is given by Eq.(2.1). Namely, Fermat's potential $\phi$ is fixed when we have determined the surface mass density $\Sigma(\vec{x})$ normalized by $\Sigma_{\text {crit }}$.

$\phi$ depends on the cosmological large scale structure through $\Sigma_{\text {crit }}$ whereas $\Sigma(\vec{x})$ does on the lens models. The former problem is, for instance, concerned with the information of inhomogeneity of the universe, Dyer-Roeder distance, cosmological parameters, etc. The latter is related with the information of lensing object.

Our arguments in this article are essentially restricted in the latter problem model problem.

As models, we may consider a variety of elliptical lens models that have different power dependence of distance in mass distribution. However their analytical surveys are not tractable and dedicated to numerical calculations. On the other hand, multipole expansion model seems to be rather tractable.

In any case, the more models are sophisticated, the more parameter space gets complicated. Parameters are, for instance, mass, finite core size, ellipticity, multipole moments and their angles, etc. Such parameters are fixed to reproduce the observed quantities such as image positions, image amplifications and time delays between several images. However such parameter fitting becomes ambiguous and best fitting may not be unique in models with many parameters. Even if parameters had been fitted uniquely, it does not mean systematic understanding of GL.

More concretely speaking, observed data are biased in many cases by complicated unknown reasons. For instance, observed image amplifications are disturbed by the dust lying between the source and observer. Also the magnitude of such disturbance may be different in the respective light path. In that case it is not necessarily reliable that we fit the parameters naively to the raw observed data. These problems are rather common to the observed cosmology. However they are especially serious in GL, since we have no confirmed physical meaning in mass distribution model of lensing objects.

So we need some strategy for searching the universal structure of GL that is irrelevant to models and boundary conditions. This is another problem - formulation problem. We apply catastrophe theory $(\mathrm{CT})$ [3] for such strategy.

The application of CT to GL has already been performed in gravitational microlensing and proved to be very useful [4]. The concepts of optical depth, the time duration and amplification of image are made clear in the framework of CT.

This paper is organized as follows. In $\S 2$ we argue general framework of singularities of caustics in complex coordinates, which is applied to the concrete models in subsequent sections. In $\S 3$ we discuss multipole expansion model. We will see how CT work in gravitational lensing. However it is indispensable to consider the transition of 
singularities analytically for that purpose. These analyses are confirmed by numerical calculations in $\S 4$ and applied to the real multiple quasar system. In the last section we consider elliptical lens model and see the consistency with the multipole expansion model. 


\section{General framework in complex coordinates}

Assuming the thin lens approximation, Fermat's potential is given by

$$
\phi(\vec{x}, \vec{y})=\frac{1}{2}(\vec{x}-\vec{y})^{2}-\frac{1}{\pi} \int d^{2} x^{\prime} \kappa\left(\vec{x}^{\prime}\right) \ln \left|\vec{x}-\vec{x}^{\prime}\right| .
$$

Here $\vec{x}$ and $\vec{y}$ are two dimensional coordinates of image and source positions, respectively, which are illustrated in Fig.1 .

Fig.1

$\kappa$ is the surface mass density of lens object,

$$
\kappa(\vec{x})=\Sigma(\vec{x}) / \Sigma_{\text {crit }},
$$

normalized by

$$
\Sigma_{c r i t} \equiv \frac{D_{O S}}{4 \pi G D_{O L} D_{L S}} .
$$

Thus we deal with the mapping of lens plane $\mathbf{R}^{\mathbf{2}}$ to source plane $\mathbf{R}^{\mathbf{2}}$.

So it becomes very convenient to introduce the complex coordinates,

$$
\left(u_{1}, u_{2}\right) \equiv(u, \bar{u}) \equiv\left(x_{1}+i x_{2}, x_{1}-i x_{2}\right) .
$$

Hereafter, we use Latin letters as the indices for real coordinates and Greek ones as the indices for complex coordinates. And we use the notation for derivatives, $\phi_{i}=\partial_{i} \phi=\partial \phi / \partial x_{i}$, etc. Then we obtain the following expressions for the various quantities used to classify the singularities of caustics [1],

$$
\begin{gathered}
D \equiv \operatorname{det}\left(\phi_{i j}\right) \equiv-4 \tilde{D}=-4 \operatorname{det}\left(\phi_{\alpha \beta}\right)=4\left(\left|\phi_{u \bar{u}}\right|^{2}-\left|\phi_{u u}\right|^{2}\right)=(1-\kappa)^{2}-|\gamma|^{2}, \\
\Delta \phi \equiv \operatorname{Tr}\left(\phi_{i j}\right)=4 \phi_{u \bar{u}}=2(1-\kappa), \\
\gamma=2 \phi_{\bar{u} \bar{u}}, \\
\Delta \equiv \operatorname{det}\left(D_{i j}\right) \equiv 16 \tilde{\Delta}=16 \operatorname{det}\left(\tilde{D}_{\alpha \beta}\right),
\end{gathered}
$$

where $\gamma$ is the shear. Critical line is a curve in image plane characterized by $D=0$ where the amplification of images $\mu \equiv \frac{1}{D}$ becomes $\infty$. Next the quantity that we often use in the classification of the singularities is the derivative operator along the critical line. 


$$
(\vec{T} \cdot \nabla)=T_{u} \partial_{u}+T_{\bar{u}} \partial_{\bar{u}}
$$

where $T_{u}$ is a tangential vector to critical line,

$$
T_{u} \equiv \frac{1}{2 i} \partial_{\bar{u}} \tilde{D}
$$

So we obtain the expression

$$
\hat{L} \equiv(\vec{T} \cdot \nabla)=\frac{1}{2 i}\left(\partial_{\bar{u}} \tilde{D} \partial_{u}-\partial_{u} \tilde{D} \partial_{\bar{u}}\right)
$$

Here we can represent the conditions for various singularities in the complex coordinates.

\subsection{Singularities of Caustics}

1. Cuspoid sequence

1-a. Fold singularity :

$$
0=4 \tilde{D}=-\left[(1-\kappa)^{2}-\left|2 \phi_{u u}\right|^{2}\right]
$$

1-b. Cusp singularity : Besides 1-a condition,

$$
0=\hat{L} \phi_{u}=\frac{1}{2 i}\left(\partial_{\bar{u}} \tilde{D} \phi_{u u}-\partial_{u} \tilde{D} \phi_{u \bar{u}}\right)
$$

1-c. Swallowtail singularity : Besides 1-b conditions,

$$
0=\hat{L}^{2} \phi_{u}
$$

1-d. Butterfly singularity : Besides 1-c conditions,

$$
0=\hat{L}^{3} \phi_{u}
$$

2. Umbilic :

$$
\phi_{\alpha \beta}=0 \quad(\alpha, \beta=u, \bar{u})
$$

2-a. Elliptic Umbilic

$$
\tilde{\Delta}>0
$$

2-b. Hyperbolic Umbilic

$$
\tilde{\Delta}<0
$$


2-c. Parabolic Umbilic

$$
\tilde{\Delta}=0
$$

3. Beak-to-beak and Lips

$$
\partial_{u} \tilde{D}=0
$$

3-a. Lips

$$
\tilde{\Delta}>0
$$

3-b. Beak-to-Beak

$$
\tilde{\Delta}<0
$$

\subsection{Shapes of images}

Fermat's potential is given by

$$
\phi=\frac{1}{2}|u-v|^{2}-\psi(u, \bar{u}),
$$

where $\psi$ is deflection potential and $v \equiv y_{1}+i y_{2}$ represents source position. Lens equation (Fermat's principle) gives

$$
v=u-2 \partial_{\bar{u}} \psi \equiv F(u, \bar{u}) .
$$

Differentiating Eq.(2.21), we obtain

$$
\begin{aligned}
d u & =\frac{1}{\left|F_{u}\right|^{2}-\left|F_{\bar{u}}\right|^{2}}\left(\bar{F}_{u} d v-F_{\bar{u}} d \bar{v}\right)=\frac{1}{D}[(1-\kappa) d v-\gamma d \bar{v}], \\
d \bar{u} & =\frac{1}{\left|F_{u}\right|^{2}-\left|F_{\bar{u}}\right|^{2}}\left(-\bar{F}_{\bar{u}} d v+F_{u} d \bar{v}\right)=\frac{1}{D}[-\bar{\gamma} d v+(1-\kappa) d \bar{v}],
\end{aligned}
$$

where

$$
F_{u} \equiv 1-2 \partial_{u} \partial_{\bar{u}} \psi=1-\kappa \quad(\text { real }),
$$

and

$$
F_{\bar{u}} \equiv-2 \partial_{\bar{u}}^{2} \psi=\gamma .
$$

Therefore Eq.(2.4) is rewritten as 


$$
D=\left|F_{u}\right|^{2}-\left|F_{\bar{u}}\right|^{2}
$$

So $|d u|^{2}$ becomes

$$
|d u|^{2}=\frac{(1-\kappa)^{2}|d v|^{2}}{D^{2}}\left[1+|\lambda|^{2}-\left(\lambda \frac{d v}{d \bar{v}}+c . c .\right)\right] .
$$

Here we have introduced the notation

$$
\lambda \equiv \bar{F}_{\bar{u}} / F_{u}=\frac{\bar{\gamma}}{1-\kappa} .
$$

From Eqs.(2.22) and (2.25), we know that the $d v$ which gives the major axis of an image satisfies,

$$
\arg \left(\lambda \frac{d v}{d \bar{v}}\right)=\pi
$$

Therefore argument of $d u$ along major axis is

$$
\begin{aligned}
\arg (d u) & =\frac{1}{2} \arg (\gamma)+\frac{1}{2} \arg (1-\kappa)+\frac{\pi}{2} & \\
& =\frac{1}{2} \arg (\gamma)+\frac{\pi}{2} \theta(1-\kappa), & (\bmod . \pi),
\end{aligned}
$$

where $\theta$ is step function. From Eq.(2.9), the vector tangential to critical line is

$$
\begin{aligned}
T_{u} & =\frac{1}{2 i} \tilde{D}_{\bar{u}} \\
& =\frac{1}{4 i}\left[\kappa_{u} \psi_{\bar{u} \bar{u}}+2 \psi_{\bar{u} \bar{u} \bar{u}} \psi_{u u}+(1-\kappa) \kappa_{\bar{u}}\right]
\end{aligned}
$$

Finally, we obtain the relative correlation between the direction of image elongation and critical curve,

$$
\arg \left(\frac{d u}{T_{u}}\right)=\frac{1}{2} \arg (\gamma)+\frac{\pi}{2} \theta(\kappa-1)-\arg \left(\tilde{D}_{\bar{u}}\right)
$$

The meaning of Eq.(2.30) is explained in the concrete models in subsequent sections. Here we show how it works in the most simple model, spherical model.

In this case, $\psi=\psi\left(|u|^{2}\right)$ and

$$
\gamma=-2 \partial_{\bar{u}}^{2} \psi=-2 u^{2} \psi^{\prime \prime} \quad \text { etc. , }
$$

where $\psi^{\prime}=\frac{d \psi}{d \omega}$ with $\omega \equiv|u|^{2}$.

So we obtain the equation,

$$
\arg (d u)=\arg u+\frac{1}{2} \arg \left(\psi^{\prime \prime}\right)+\frac{\pi}{2} \theta(\kappa-1) .
$$


If we assume that $\kappa$ is monotonically decreasing function of $\omega$, we obtain the inequality,

$$
\begin{aligned}
\psi^{\prime \prime} & =\frac{1}{\omega}\left(\frac{\kappa}{2}-\psi^{\prime}\right) \\
& =\frac{1}{2 \omega^{2}}\left[\omega \kappa-\int_{0}^{\omega} d \omega \kappa\right]<0 .
\end{aligned}
$$

Therefore the images are elongated along

$$
\left\langle\begin{array}{l}
\text { circular direction for } \kappa-1<0 \\
\text { radial direction for } \kappa-1>0
\end{array}\right.
$$

This conclusion is irrelevant to the image position relative to the critical lines. 


\section{Multipole Expansion}

Multipole expansion is the expansion of Eq.(2.1) in powers of $\frac{1}{|\vec{x}|}$ and is given by

$$
\phi=\frac{1}{2}(\vec{x}-\vec{y})^{2}-\frac{m}{2} \ln |\vec{x}|^{2}+\frac{\vec{x} \cdot \vec{d}}{|\vec{x}|^{2}}+\frac{{ }^{t} \vec{x} \hat{q} \vec{x}}{|\vec{x}|^{4}},
$$

where $m, \vec{d}, \hat{q}$ are reduced total mass, dipole and quadrupole moments of lens object, respectively. The "codimension" is identical with the number of coordinates independent equalities. And the condition whether the singularity is generic or not is determined by Dimension of parameter space $\geq$ Codimension. State variables $\vec{x}$ and control parameters $\vec{y}, m, \vec{d}, \hat{q}$ are expressed in complex coordinates as

$$
\begin{array}{ll}
z=x_{1}+i x_{2}, & s=y_{1}+i y_{2}, \\
d=d_{1}+i d_{2}, & q=q_{11}+i q_{12} \quad\left(q_{11}+q_{22}=0\right) .
\end{array}
$$

Taking, furthermore, the following rescaling,

$$
u=\frac{z}{m^{\frac{1}{2}}}, \quad v=\frac{s}{m^{\frac{1}{2}}}, \quad \delta=\frac{d}{m^{\frac{3}{2}}}, \quad Q=\frac{q}{m^{2}},
$$

we get Fermat's potential in terms of complex coordinates [5].

$$
\bar{\phi}=\frac{\phi}{m}=\frac{1}{2}|u-v|^{2}-\frac{1}{2} \ln |\bar{u} u|+\frac{1}{2}\left(\frac{\delta}{u}+\frac{\bar{\delta}}{\bar{u}}\right)+\frac{1}{2}\left(\frac{Q}{u^{2}}+\frac{\bar{Q}}{\bar{u}^{2}}\right) .
$$

The parameters in Eq.(3.4) are associated to physical parameters as follows:

$$
\begin{aligned}
m & =\frac{M}{M_{\text {crit }}}, \quad M_{c r i t} \equiv \frac{D_{O L} D_{O S}}{4 G D_{L S}} \\
\delta & =\frac{D_{1}+i D_{2}}{M_{\text {crit }} D_{O L}} m^{-\frac{3}{2}} \\
Q & =\frac{I_{11}+i I_{12}}{M_{\text {crit }} D_{O L}^{2}} m^{-2}
\end{aligned}
$$

where $M, D_{\nu}$ and $I_{\nu \lambda}$ correspond the physical total mass, the physical 3-dimensional dipole moment and the physical 3-dimensional quadrupole moment, respectively. One of the aims of this article to find the relationships between model parameters and singularities in analytical ways. So we first discuss about the simplified model and the lowest singularities. Our task here is to express the equalities in $\S 2$ by the multipole parameters.

Critical point condition Eq.(2.11) becomes

$$
|u|^{2}=\left|1+2 \frac{\delta}{u}+6 \frac{Q}{u^{2}}\right|
$$


Cusp condition Eq.(2.12) is

$$
\frac{\left(1+2 \frac{\delta}{u}+6 \frac{Q}{u^{2}}\right)^{3}}{\left(1+3 \frac{\delta}{u}+12 \frac{Q}{u^{2}}\right)^{2}}=\frac{|u|^{6}}{\left|1+3 \frac{\delta}{u}+12 \frac{Q}{u^{2}}\right|^{2}} .
$$

Eqs.(3.8) and (3.9) are too complicated for analytical surveys. So we discuss $\delta$ and $Q$ separately in the following.

\section{1 $\mathrm{Q}=0 \mathrm{CASE}$}

At first, we consider $\mathrm{Q}=0$ case. Then Eqs.(3.8) and (3.9) are reduced to

$$
\begin{gathered}
|u|^{2}=\left|1+2 \frac{\delta}{u}\right|, \\
\frac{\left(1+2 \frac{\delta}{u}\right)^{3}}{\left(1+3 \frac{\delta}{u}\right)^{2}}=\frac{|u|^{6}}{\left|1+3 \frac{\delta}{u}\right|^{2}} .
\end{gathered}
$$

From Eq.(3.11), we obtain

$$
\begin{gathered}
\operatorname{Im}\left[\frac{\left(1+2 \frac{\delta}{u}\right)^{3}}{\left(1+3 \frac{\delta}{u}\right)^{2}}\right]=0, \\
\frac{\left(1+2 \frac{\delta}{u}\right)^{3}}{\left(1+3 \frac{\delta}{u}\right)^{2}}>0 .
\end{gathered}
$$

We introduce the geometrical variables,

$$
\frac{u}{\delta} \equiv \frac{\tau}{|\delta|} e^{i \theta} \equiv t e^{i \theta},
$$

where $\tau$ is real and $\theta$ is the physical angle of image position from the dipole

direction. In terms of the geometrical variables, Eqs.(3.10), (3.12) and (3.13) are expressed by

$$
\begin{gathered}
|\delta|^{2} t^{3}=\sqrt{t^{2}+4 t \cos \theta+4}, \\
\sin \theta\left[3 t^{3} \cos \theta+t^{2}\left(5+16 \cos ^{2} \theta\right)+48 t \cos \theta+36\right]=0, \\
t^{5}+12 t^{4} \cos \theta+3 t^{3}\left(5+14 \cos ^{2} \theta\right)+2 t^{2} \cos \theta\left(51+16 \cos ^{2} \theta\right) \\
+12 t\left(5+8 \cos ^{2} \theta\right)+72 \cos \theta>0,
\end{gathered}
$$

respectively.

(i) $\sin \theta=0$ 
At first, we consider the critical points on the axis of the dipole direction. Then Eq.(3.16) vanishes. And Eqs.(3.10) and (3.17) are written as follows:

$$
\begin{gathered}
|\delta|^{4} y^{6}=(y+2)^{2}, \\
y(y+2)>0,
\end{gathered}
$$

where $y \equiv t \cos \theta$. The solutions of Eq.(3.19) change at $|\delta|=\sqrt{3} / 9$ qualitatively. At this value, beak-to-beak singularity appears. When $|\delta|$ is less than this value, cusp singularity appears. And when $|\delta|$ is more than this value, fold singularity appears.

(ii) $\sin \theta \neq 0$

Off the axis, we regard Eqs.(3.15)-(3.17) as the equations for $\delta, t \cos \theta(\equiv y)$ and $t^{2}(\equiv x)$. Using $x$ and $y$, Eqs.(3.15) and (3.16) become

$$
|\delta|^{4} x^{3}-x-4=4 y
$$

and

$$
x=-\frac{4(2 y+3)^{2}}{3 y+5},
$$

respectively. However, $x>0, x \geq y^{2}$ by definition, which implies

$$
-3 \leq y<-\frac{5}{3}
$$

Also Eq.(3.17) is rewritten as

$$
0<\frac{4 x}{t} \frac{(y+2)^{3}(y+3)}{(3 y+5)^{2}} .
$$

Consequently $y$ satisfies

$$
-2<y<-\frac{5}{3}
$$

Eliminating $x$ from Eqs.(3.20) and (3.21), we obtain

$$
4|\delta|^{2}(2 y+3)^{3}=(3 y+5)|y+2| .
$$

That is,

$$
|\delta|^{2}=\frac{9}{4} \frac{\rho(1-\rho)}{(2 \rho+1)^{3}} \equiv f(\rho)
$$

where $\rho$ is bounded

$$
0<\rho \equiv-(3 t \cos \theta+5)<1 .
$$

The function $f(\rho)$ relation is plotted in Fig.2. 
Fig.2

Two solutions coalesce at $|\delta|^{2}=\frac{10+7 \sqrt{7}}{6^{3}}$ in which higher singularity, swallowtail, appears. At $|\delta|$ more (less) than this value, cusp (fold) singularity appears.

\section{$3.2 \delta=0$ CASE}

Next, we consider $\delta=0$ case. Then Eqs.(3.8) and (3.9) are

$$
\begin{gathered}
|u|^{2}=\left|1+6 \frac{Q}{u^{2}}\right|, \\
\frac{\left(1+6 \frac{Q}{u^{2}}\right)^{3}}{\left(1+12 \frac{Q}{u^{2}}\right)^{2}}=\frac{|u|^{6}}{\left|1+12 \frac{Q}{u^{2}}\right|^{2}} .
\end{gathered}
$$

From Eq.(3.27) we obtain

$$
\begin{gathered}
\operatorname{Im}\left[\frac{\left(1+6 \frac{Q}{u^{2}}\right)^{3}}{\left(1+12 \frac{Q}{u^{2}}\right)^{2}}\right]=0, \\
\frac{\left(1+6 \frac{Q}{u^{2}}\right)^{3}}{\left(1+12 \frac{Q}{u^{2}}\right)^{2}}>0 .
\end{gathered}
$$

We introduce the geometrical variables

$$
\frac{u^{2}}{Q}=\frac{\tau^{2}}{|Q|} e^{2 i \theta} \equiv w e^{2 i \theta},
$$

where $\tau$ is real and $\theta$ is the physical angle of image position from the quadrupole

direction. In terms of the geometrical variables, Eqs.(3.26),(3.28) and (3.29) are expressed by

$$
\begin{gathered}
|Q| w^{2}=\sqrt{w^{2}+12 w \cos 2 \theta+36} \\
\sin 2 \theta\left[w^{4}+12 w^{3} \cos 2 \theta-36 w^{2}\left(4 \cos ^{2} 2 \theta-1\right)\right. \\
\left.-8 \cdot 6^{3} w \cos 2 \theta-4 \cdot 6^{4}\right]=0 \\
w^{6}+42 w^{5} \cos 2 \theta+36 w^{4}\left(5+14 \cos ^{2} 2 \theta\right)+6^{3} w^{3} \cos 2 \theta\left(21+4 \cos ^{2} 2 \theta\right) \\
+8 \cdot 6^{4} w^{2}\left(1+\cos ^{2} 2 \theta\right)+4 \cdot 6^{5} w \cos 2 \theta>0
\end{gathered}
$$

respectively. 
(i) $\sin 2 \theta=0$

At first, we consider the singularity on the axis of the quadrupole direction and on the axis normal to it. Then Eqs.(3.31) and (3.33) are written as follows:

$$
\begin{gathered}
|Q|^{2} y^{4}=(y+6)^{2}, \\
y(y+6)>0,
\end{gathered}
$$

where $y \equiv|u / Q|^{2} \cos 2 \theta$. The solutions of Eqs. (3.34) change at $|Q|=1 / 24$ qualitatively. At this value, beak-to-beak singularity appears. When $|Q|$ is less than this value, cusp singularity appears. And when $|Q|$ is more than this value, fold singularity appears.

(ii) $\sin 2 \theta \neq 0$

we regard Eqs.(3.31) - (3.33) at points off those axes as the equations for $Q, w \cos 2 \theta$

and $w^{2}$. Those solutions of Eqs. (3.31) - (3.33) qualitatively change at $|Q|=\frac{\sqrt{598+82 \sqrt{41}}}{192}$ and $|Q|=1 / 8$. They correspond to swallowtail and butterfly singularity, respectively. When $|Q|$ is less than the swallowtail-value, cusp singularity appears. And when $|Q|$ is more than that value, fold singularity appears. Talking about butterfly singularity, when $|Q|$ is not butterfly-value, cusp singularity always appears. These results are confirmed by numerical calculations.

\subsection{Shapes of images}

From Eq.(2.9) the vector tangential to critical line is

$$
T_{u}=\frac{1}{2 i} \psi_{u u} \psi_{\bar{u} \bar{u} \bar{u}}=\frac{1}{8 i} \bar{\gamma} \partial_{\bar{u}} \gamma
$$

for non-transparent lens model like multipole expansion. From Eqs.(3.35) and (2.30) it follows

$$
\begin{aligned}
\arg \left(\frac{d u}{T_{u}}\right) & =\frac{3}{2} \arg (\gamma)-\arg \left(\partial_{\bar{u}} \gamma\right) \\
& =\frac{\pi}{2}+\frac{1}{2} \arg \left[\frac{\left(\psi_{\bar{u} \bar{u}}\right)^{3}}{\left(\psi_{\bar{u} \bar{u} \bar{u}}\right)^{2}}\right]
\end{aligned}
$$

If source position is in the neighborhood of cusp singularity, $\frac{\left(\psi_{\bar{u}} \bar{u}\right)^{3}}{\left(\psi_{\bar{u}} \bar{u}\right)^{2}}$ is concluded to be almost negative definite from Eq.(3.9). Therefore,

$$
\arg \left(\frac{d u}{T_{u}}\right) \sim 0 \text { or } \pi \text {. }
$$

Namely, the images in the neighborhood of cusp are parallel to critical line. 
For the general source position,

$$
\arg \left(\frac{d u}{T_{u}}\right)=\frac{3}{2} \arg \left[1+2 \frac{\bar{\delta}}{\bar{u}}+6 \frac{\bar{Q}}{\bar{u}^{2}}\right]-\arg \left[1+3 \frac{\bar{\delta}}{\bar{u}}+12 \frac{\bar{Q}}{\bar{u}^{2}}\right]
$$




\section{Numerical Calculations}

In the previous section we have analyzed lens equation in which lensing object is modeled by multipole expansion. In this section we will check the results of each case by numerical calculations in the first two subsections. In the last subsection we will apply multipole expansion model to PG1115+080 [6], which is compared with the article by Yoshida and Omote [5].

\section{1 $\mathrm{Q}=0$ case}

Fig. 3 shows the behaviors of caustics and critical lines in the neighborhood of $|\delta|=\frac{\sqrt{3}}{9}$ at which beak-to-beak appears on axis.

Fig.3

In $|\delta|<\frac{\sqrt{3}}{9}$, the inequality in Eqs.(3.19) is satisfied and cusp appears on axis (Fig.3a). At $|\delta|=\frac{\sqrt{3}}{9}$ cusps coalesce to beak-to-beak on axis (Fig.3b). In $|\delta|>\frac{\sqrt{3}}{9}$, the inequality in Eqs.(3.19) is not satisfied. So cusps disappear and fold is born (Fig.3c).

Fig. 4 shows the behaviors in the neighborhood of $|\delta|^{2}=\frac{10+7 \sqrt{7}}{6^{3}}$ at which swallowtail appears off axis.

Fig.4

This corresponds to the argument in subsection $§ 3.1($ ii). As was indicated there, cusp singuralities at points P change to swallowtail when $|\delta|$ increases to $\delta_{c} \equiv \sqrt{\frac{10+7 \sqrt{7}}{6^{3}}}$ (Fig.4a and Fig.4b) and cusps disappear and fold appears(Fig.4c). Swallowtail singularity is easily checked by Fig.4a corresponding to $|\delta|<\delta_{c}$.

\section{$4.2|\delta|=0$ Case}

First we simulate the case (i) in $\S 3-2$. Fig. 5 shows the behaviors of singularities in the neighborhood of $|Q|=\frac{1}{24}$ at which beak-to-beak appears on $\theta=\frac{\pi}{2}$ axis.

Fig.5 
At $|Q|<\frac{1}{24}$, the inequality in Eqs. (3.34) is satisfied and cusps appear (Fig.5a). They coalesce to beak-to-beak at $|Q|=\frac{1}{24}$ on the $Y$-axis (Fig.5b). The inequality in Eqs. (3.34) is not satisfied at $|Q|>\frac{1}{24}$ and fold appears instead of cusp (Fig.5c).

Furthermore the case (ii) is simulated in Fig.6 and Fig.7.

Fig.6

Fig.7

$|Q|$ increases passing through two critical points, $\frac{1}{8}\left(\equiv Q_{1}\right)$ and $\frac{\sqrt{598+82 \sqrt{41}}}{192}\left(\equiv Q_{2}\right)$. Fig.6b and Fig.7b correspond to the transition points of butterfly and swallowtail, respectively. This is confirmed from Fig.6c and Fig.7a that are diagrams with $|Q|>$ $Q_{1}$ and $|Q|<Q_{2}$, respectively.

\subsection{Application to multiple quasar}

In this subsection we apply multipole expansion model Eq.(3.4) to lensing system PG1115+080. We adopt the observed data by Christian et al [7], which is exhibited in Table 1.

\section{Table 1}

Numerical result is shown in Fig.8.

Fig. 8

Here we have taken the values for control parameters in Eq.(3.4) as follows:

$$
\begin{gathered}
\text { source position }=\left(0^{\prime \prime} .009,0^{\prime \prime} .021\right), \\
m=3.468 \times 10^{-11}, \\
|\delta|=0.1912, \quad \theta_{\delta}=91^{\circ} .85, \\
|Q|=0.0864, \quad \theta_{Q}=135^{\circ} .79,
\end{gathered}
$$


where $\theta_{\delta}$ and $\theta_{Q}$ are arguments of $\delta$ and $Q$, respectively. The parameters adopted in [5] are related to our parameters by

$$
\begin{aligned}
\mu & =m(\text { mass }) \\
|\delta| & =m^{1 / 2}\left|\delta_{\text {our }}\right|=1.126 \times 10^{-6}, \\
|\xi| & =2 m|Q|=5.993 \times 10^{-12}, \\
\chi_{d} & =\theta_{\delta}-\frac{\theta_{Q}}{2}=23^{\circ} .95, \\
\chi_{G} & =\frac{\theta_{Q}}{2}=67^{\circ} .90 .
\end{aligned}
$$

Calculated image positions are

$$
\begin{aligned}
\theta\left(A_{1}\right) & =\left(-0^{\prime \prime} .84,-0^{\prime \prime} .87\right) \\
\theta\left(A_{2}\right) & =\left(-1^{\prime \prime} .12,-0^{\prime \prime} .26\right) \\
\theta(B) & =\left(0^{\prime \prime} .70,-0^{\prime \prime} .60\right) \\
\theta(C) & =\left(0^{\prime \prime} .35,1^{\prime \prime} .34\right) \\
\theta(D) & =\left(0^{\prime \prime} .56,-0^{\prime \prime} .42\right) \\
\theta(E) & =\left(-0^{\prime \prime} .49,0^{\prime \prime} .08\right)
\end{aligned}
$$

where we had the additional two images labeled by $\mathrm{D}$ and $\mathrm{E}$ as was indicated by Ref. [5]. Here we show source position and image positions $\theta$ in unnormalized values that can be compared with observed values, while we use variables $u, v$ normalized by $\sqrt{m}$ in Eq.(3.4).

The relative magnifications of images are also calculated from Eq.(2.4) as

$$
\begin{aligned}
& \left|\frac{\mu_{A_{1}}}{\mu_{C}}\right|=2.55, \quad\left|\frac{\mu_{A_{2}}}{\mu_{C}}\right|=2.22, \quad\left|\frac{\mu_{B}}{\mu_{C}}\right|=0.78, \\
& \left|\frac{\mu_{D}}{\mu_{C}}\right|=0.15, \quad\left|\frac{\mu_{E}}{\mu_{C}}\right|=0.73 \times 10^{-2} .
\end{aligned}
$$

We will check the validity of approximation in multipole expansion. It must hold the following conditions

$$
1 \gg \frac{|\delta|}{|u|} \gg \frac{|Q|}{|u|^{2}}
$$

The best fit parameters give

$$
\frac{|\delta|}{|u|} \approx 0.2266 \text { and } \frac{|Q|}{|u|^{2}} \approx 0.1390
$$

Thus the condition Eq.(4.5) is rather marginal. So we should check that $2^{3}$-pole and higher multipole terms less contribute, though we do not touch this problem in this paper. 


\section{Discussions}

We have argued the classification of singularities of caustics based on the multipole expansion model of lensing object. In the application to the multiple quasar system of PG1115+080 there have arisen several problems. Fig.8 shows that deamplified images $\mathrm{D}$ and $\mathrm{E}$ are both normal to critical line. $\operatorname{Arg}\left(\frac{d u}{T_{u}}\right)$ is given by

$$
\arg \left(\frac{d u}{T_{u}}\right)=\frac{1}{2} \arg (\gamma)-\arg \left(\bar{\gamma} \partial_{\bar{u}} \gamma\right)
$$
by

from Eq.(2.30) and $\kappa=0$ for multipole expansion. Whereas amplification is given

$$
\mu \equiv D^{-1}=\frac{1}{1-|\gamma|^{2}}
$$

Amplification does not depend on the argument of $\gamma$.

Therefore if we are concerned with fold catastrophe we can not relate the amplification with the image direction relative to the critical line.

We should also be careful to the validity of approximation. Image D and E are near to the lens position and multipole expansion is badly convergent. We pointed out that dipole and quadrupole terms give the contribution of same order.

It is certainly desirable to consider the same lensing system based on the several models and to check the consistency of models.

We will compare our results with those of elliptical lens model by Narasimha et al [8]. Lensing object in [8] is modeled as the oblate spheroid

$$
x^{2}+y^{2}+\frac{z^{2}}{1-e^{2}}=a^{2} .
$$

Mass density is given by

$$
\begin{aligned}
\rho(a) & =\rho_{0}\left(1+\frac{a^{2}}{r_{0}^{2}}\right)^{-\frac{k}{2}} & & \text { for } a / r_{c} \leq n \\
& =0 & & \text { for } a / r_{c}>n
\end{aligned}
$$

where $r_{c}$ is core size. We can always set $x, y$ coordinates in lens plane and z-axis is tilted relative to the photon path by an angle $\varphi$.

Then the surface mass density projected on the lens plane is

$$
\Sigma(b)=\sqrt{\frac{1-e^{2}}{1-e^{2} \sin ^{2} \varphi}} K_{k}
$$

where

$$
K_{k}(b)=\int_{b^{2} / r_{c}^{2}}^{n^{2}} d \omega \frac{\rho_{0} r_{c}}{\sqrt{\omega-\left(\frac{b}{r_{c}}\right)^{2}}}(1+\omega)^{-\frac{k}{2}}
$$


and

$$
b^{2}=x^{2}+\frac{y^{2}}{1-e^{2} \sin ^{2} \varphi} .
$$

In this model the dipole component, $\delta$, vanishes and we will estimate the quadrupole component, $\xi$, adopted in [5] as

$$
\begin{aligned}
\xi & =\frac{1}{D_{O L}^{2}} \frac{\int d \vec{x} \Sigma(\vec{x}) z^{2}}{\int d \vec{x} \Sigma(\vec{x})} \\
& =\frac{e^{2} \sin ^{2} \varphi}{2 D_{O L}^{2}} \frac{\int_{0}^{n r_{c}} d b b^{3} K_{k}(b)}{\int_{0}^{n r_{c}} d b b K_{k}(b)} \\
& \equiv \frac{e^{2} \sin ^{2} \varphi}{2} \frac{r_{c}^{2}}{D_{O L}^{2}} \frac{F_{k}^{(1)}}{F_{k}^{(0)}}
\end{aligned}
$$

where $z$ is given by Eq.(3.2) and where $F_{k}^{(l)}$ is defined by

$$
\begin{aligned}
F_{k}^{(l)} & \equiv \int_{0}^{n^{2}} d x x^{l} \int_{x}^{n^{2}} d y \frac{1}{\sqrt{y-x}}(1+y)^{-\frac{k}{2}} \\
& =\int_{0}^{n^{2}} d x x^{l}(1+x)^{\frac{1-k}{2}} \int_{0}^{\sqrt{\frac{n^{2}-x}{1+x}}} d u\left(1+u^{2}\right)^{-\frac{k}{2}}
\end{aligned}
$$

As is easily checked

$$
F_{3}^{(0)}=2\left[\ln \left(\sqrt{n^{2}+1}+n\right)-\frac{n}{\sqrt{n^{2}+1}}\right]
$$

and

$$
F_{3}^{(0)}+F_{3}^{(1)}=\frac{2}{3} \frac{n^{3}}{\sqrt{n^{2}+1}}
$$

Therefore we obtain

$$
\frac{F_{3}^{(1)}}{F_{3}^{(0)}} \simeq \begin{cases}48.5 & \text { for } \mathrm{n}=20 \\ 95.9 & \text { for } \mathrm{n}=30\end{cases}
$$

$r_{c} / D_{O L}$ in Eq.(5.8) is related to the numerical result by

$$
\frac{r_{c}}{D_{O L}}=\frac{\theta(\mathrm{rad})}{s}
$$

where $\theta(\mathrm{rad})$ is the observed image separation angle in unit of radian and $s$ is the separation length in unit of core size.

So 


$$
\xi=\frac{e^{2} \sin ^{2} \varphi}{2}\left(\frac{\theta(\mathrm{sec})}{s}\right)^{2} \frac{F_{k}^{(1)}}{F_{k}^{(0)}} \times 2.4 \times 10^{-11}
$$

We adopt the values from Table 1 in 8

$$
k=3, \quad e \sin \varphi=0.6, \quad n=20, \quad \frac{4 G M D}{r_{c}^{2} c^{2}}=32, \quad \text { and } \quad s_{B C}=7.46,
$$

where $s_{B C}$ is $s$ between images B and C. Combining these values with $\theta_{B C} \simeq 1^{\prime \prime} .989$ we obtain

$$
\xi=1.51 \times 10^{-11} .
$$

If we adopt other values $\xi$ takes the range

$$
\xi_{\text {elliptical }} \simeq(2 \sim 4) \times \xi_{\text {multipole }} .
$$

This seems that quadrupole moments evaluated by the different models coincide in this order.

We may set $k=1$ or $k=5$ in elliptical lens model. Especially $k=2$ seems to be important for isothermal model [9] [10]. In this case, numerical calculation becomes cumbersome and will be discussed elsewhere. Lens models so far considered are somehow empirical. By applying the several models into the same lensing system, we can construct the more reliable model of lensing object and gravitational lensing.

We could not still exhaust the merit of CT in this article. In the near future, the numbers of observed lensing events will rapidly increase and we will get more precise information [11]. CT will play increasingly essential role in that situation. 


\section{Acknowledgements}

We have benefited greatly from discussions and communications with Professors H.Yoshida, M.Omote and K.Tomita and Dr. N.Makino.

\section{References}

[1] P. Schneider, J. Ehlers and E. E. Falco, Gravitational Lenses (Springer-Verlag, 1992).

[2] R. Blandford and C. S. Kochanek, "Gravitatinal Lenses" in Dark Matters in the Universe, ed. by J. Bahcall, T. Piran and S. Weinberg (World Scientific, 1987).

[3] V. I. Arnol'd, Catastrophe Theory (Springer-Verlag, 1992).

[4] K. Chang and S. Refsdal, Nature 282, 561 (1979).

B. Paczynski, Ap. J. 304, 1 (1986).

[5] H. Yoshida and M. Omote, Prog. Theor. Phys. 79, 1095 (1988).

[6] D. Walsh, R. F. Carswell and R. J. Weymann, Nature 279, 381 (1979).

R. J. Weymann, D.Latham, J. R. P. Angel, R. F. Green, J. W. Liebert, D. E. Turnshek and J. A. Tyson, Nature 285, 641 (1980).

[7] C. A. Christian, D. Crabtree and P. Waddell, Ap. J. 312, 45 (1987).

[8] D. Narasimha, K. Subramanian and S. M. Chitre, Mon. Not. R. Astr. Soc. 200, 941 (1982).

[9] P.Kormann, P.Schneider and M.Bartelmann, Astron. Astrophys. 284, 285 (1994) and references therein.

[10] N. Makino and K. Tomita, Publ. Astron. Soc. Japan 47, 117 (1995).

[11] See for instanse, "Digital Sky Survey of the Northern Galactic Cap," ed. by J. E. Gunn (Princeton Univ., 1994) 


\section{Figure Captions}

Fig.1 Schematic diagram of the gravitational lens geometry. The solid line shows the real path of the light ray.

Fig.2 Graph of the function $f(\rho)$ given in Eq.(3.25). Two solutions of $f(\rho)=|\delta|^{2}$ coalesce at $|\delta|^{2}=\frac{10+7 \sqrt{7}}{6^{3}}$, maximum of $f(\rho)$.

Fig.3 Caustic and critical lines in neighborhood of $|\delta|=\sqrt{3} / 9$ at which beak-to-beak appears on axis.

Fig.3a $|\delta|=0.17$

Fig.3b $|\delta|=\sqrt{3} / 9 \sim 0.19$

Fig.3c $|\delta|=0.21$

Fig.4 Caustic and critical lines in neighborhood of $|\delta|=\delta_{c}=\frac{\sqrt{10+7 \sqrt{7}}}{6 \sqrt{6}}$ at which swallowtail appears. Points $\mathrm{P}$ in Fig.4a are the points of cusp singularities which change to swallowtail when $|\delta|$ increases.

Fig.4a $|\delta|=0.32$

Fig.4b $|\delta|=\delta_{c} \sim 0.36$

Fig.4c $|\delta|=0.50$

Fig.5 Caustic and critical lines in neighborhood of $|Q|=1 / 24$ at which beak-to-beak appears on $Y$-axis.

Fig.5a $|Q|=0.03$

Fig.5b $|Q|=1 / 24 \sim 0.04$

Fig.5c $|Q|=0.05$

Fig.6 Caustic and critical lines in neighborhood of $|Q|=Q_{1}=1 / 8$ at which butterfly appears. Point $\mathrm{P}$ in Fig.6b is the point where butterfly appears.

Fig.6a $|Q|=0.12$

Fig.6b $|Q|=1 / 8$

Fig.6c $|Q|=0.13$

Fig.7 Caustic and critical lines in neighborhood of $|Q|=Q_{2}=\frac{\sqrt{598+82 \sqrt{41}}}{192}$ at which swallowtail appears.

Fig.7a $|Q|=0.17$

Fig.7b $|Q|=Q_{2} \sim 0.1745$ 
Fig.7c $|Q|=0.18$

Fig.8 Shapes of the images, critical and caustic line for the multiple quasar PG1115+080 from our calculation. The lensing galaxy is at coordinate origin. Observed image positions are denoted by crosses.

Table 1 Observed image positions and their amplifications of PG1115+080. 


\begin{tabular}{|c|r|r|l|}
\hline image & $x_{1}(\mathrm{sec})$ & $x_{2}(\mathrm{sec})$ & $\mu / \mu_{C}$ \\
\hline$A_{1}$ & $-1^{\prime \prime} .27$ & $-2^{\prime \prime} .08$ & 3.22 \\
\hline$A_{2}$ & $-1^{\prime \prime} .44$ & $-1^{\prime \prime} .62$ & 2.49 \\
\hline$B$ & $0^{\prime \prime} .39$ & $-1^{\prime \prime} .95$ & 0.64 \\
\hline$C$ & $0^{\prime \prime} .00$ & $0^{\prime \prime} .00$ & 1.00 \\
\hline \hline lens & $-0^{\prime \prime} .33$ & $-1^{\prime \prime} .35$ & \\
\hline
\end{tabular}

Table 1: Observational data 


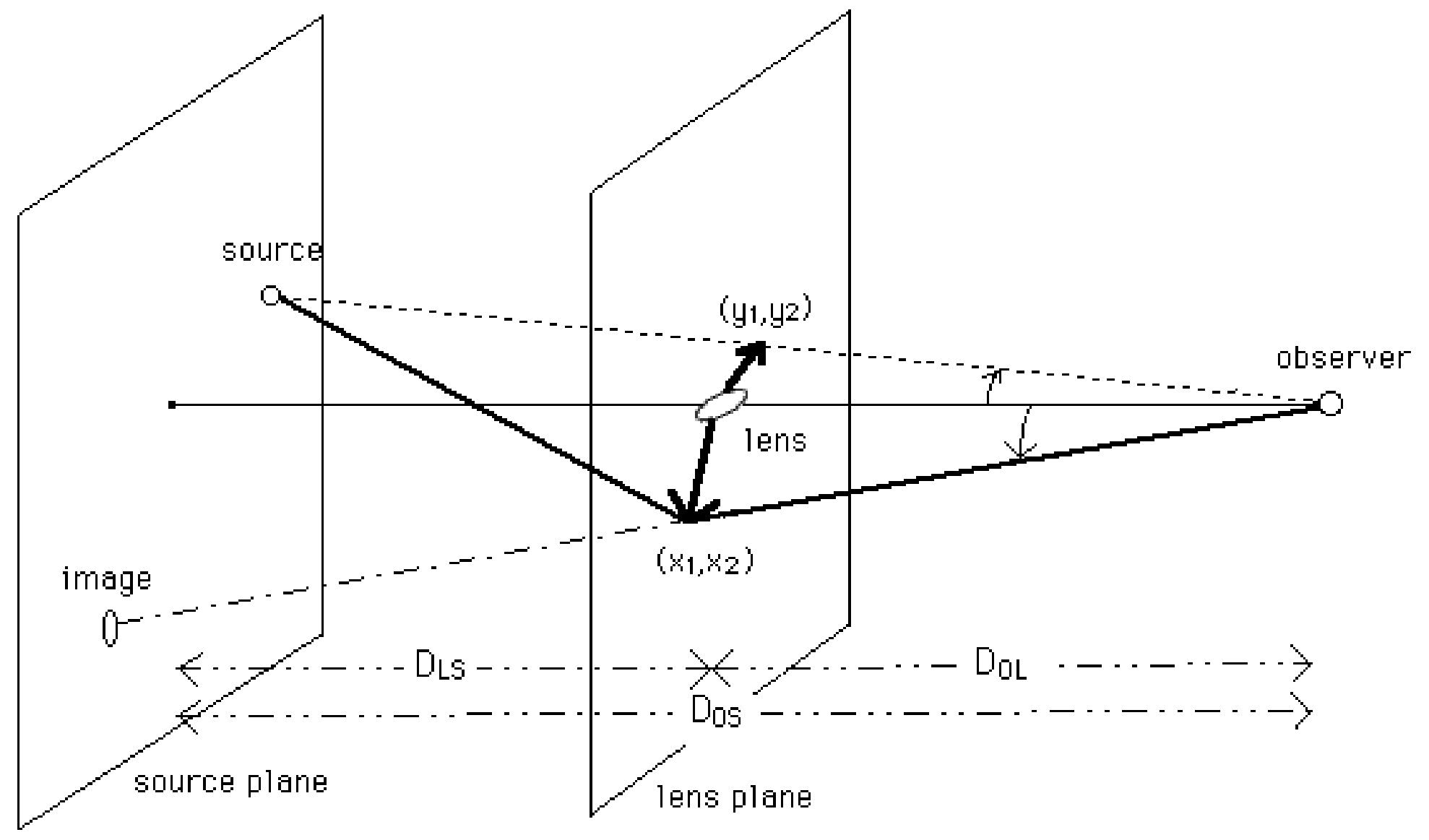

Fig. 1 
Fig. 2

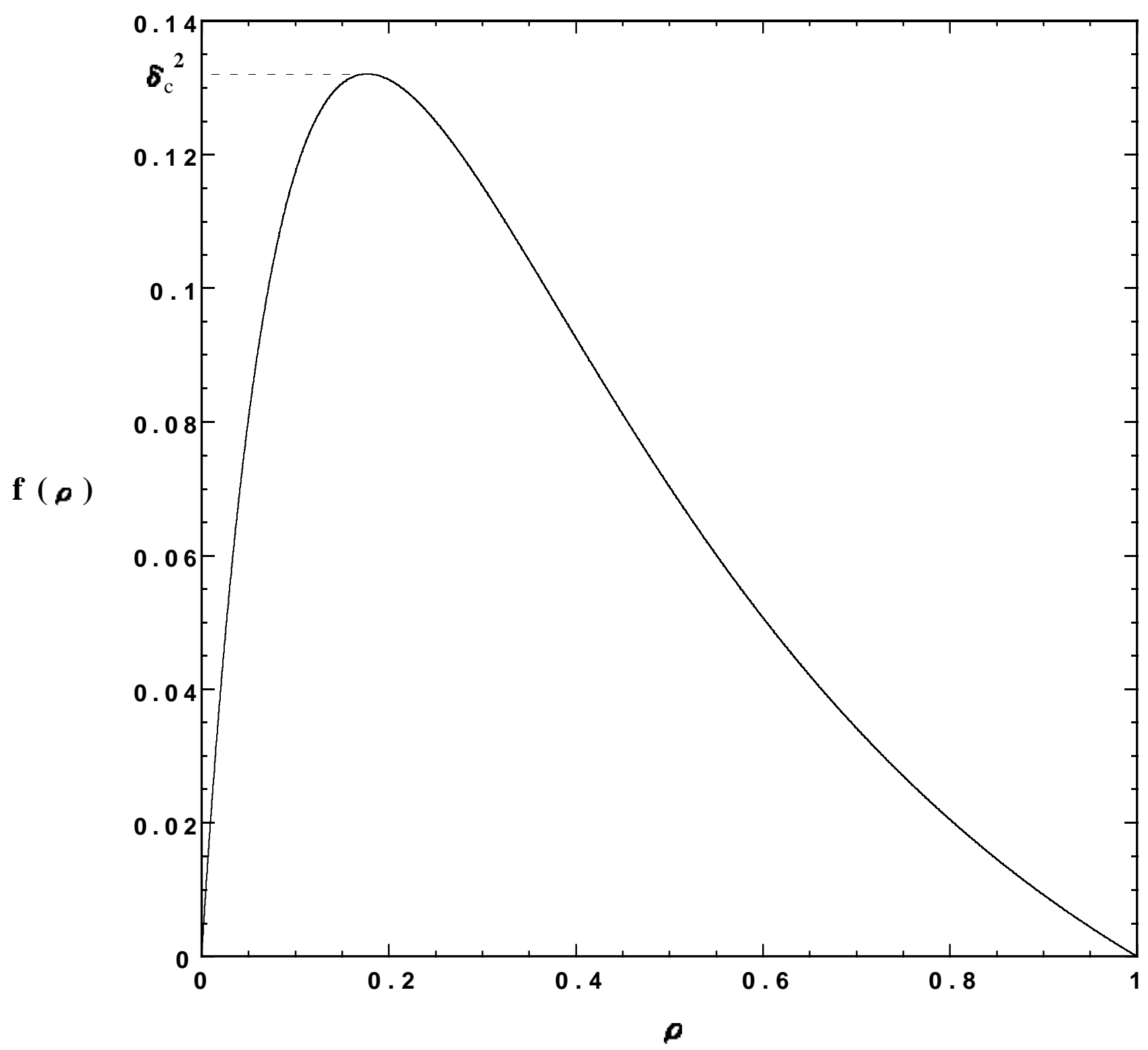


Fig. 3a

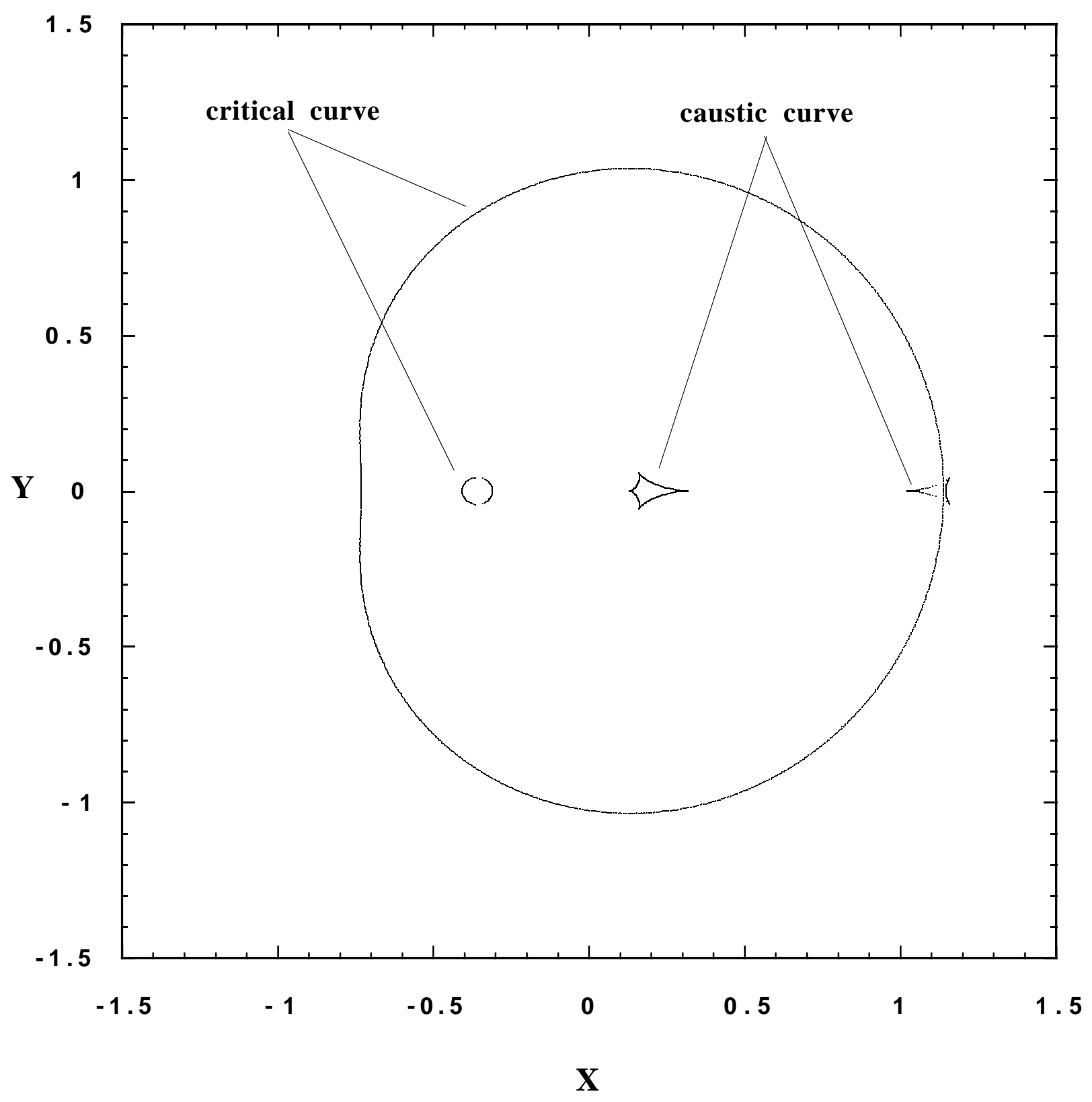


Fig. 3b

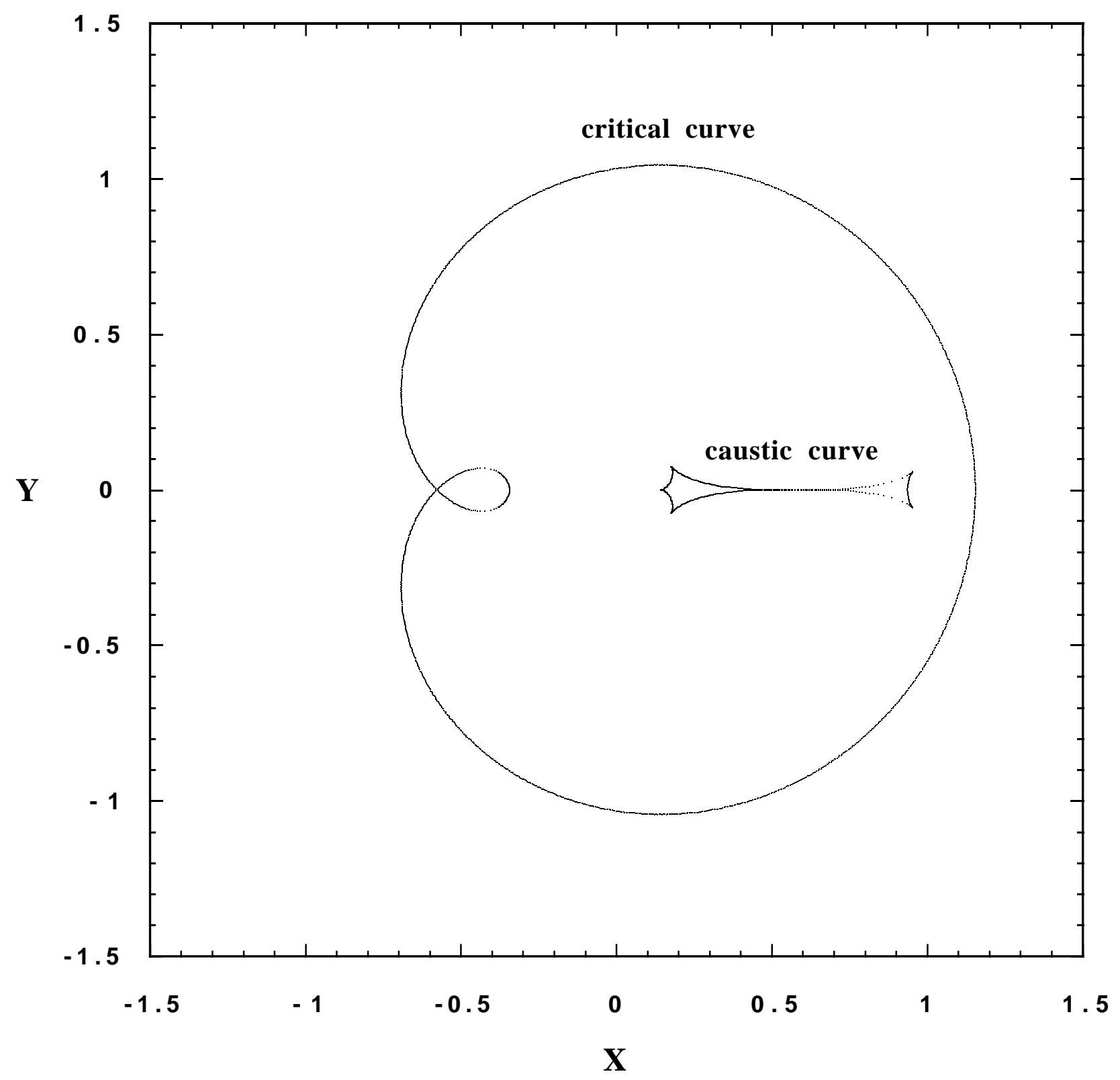


Fig. 3c

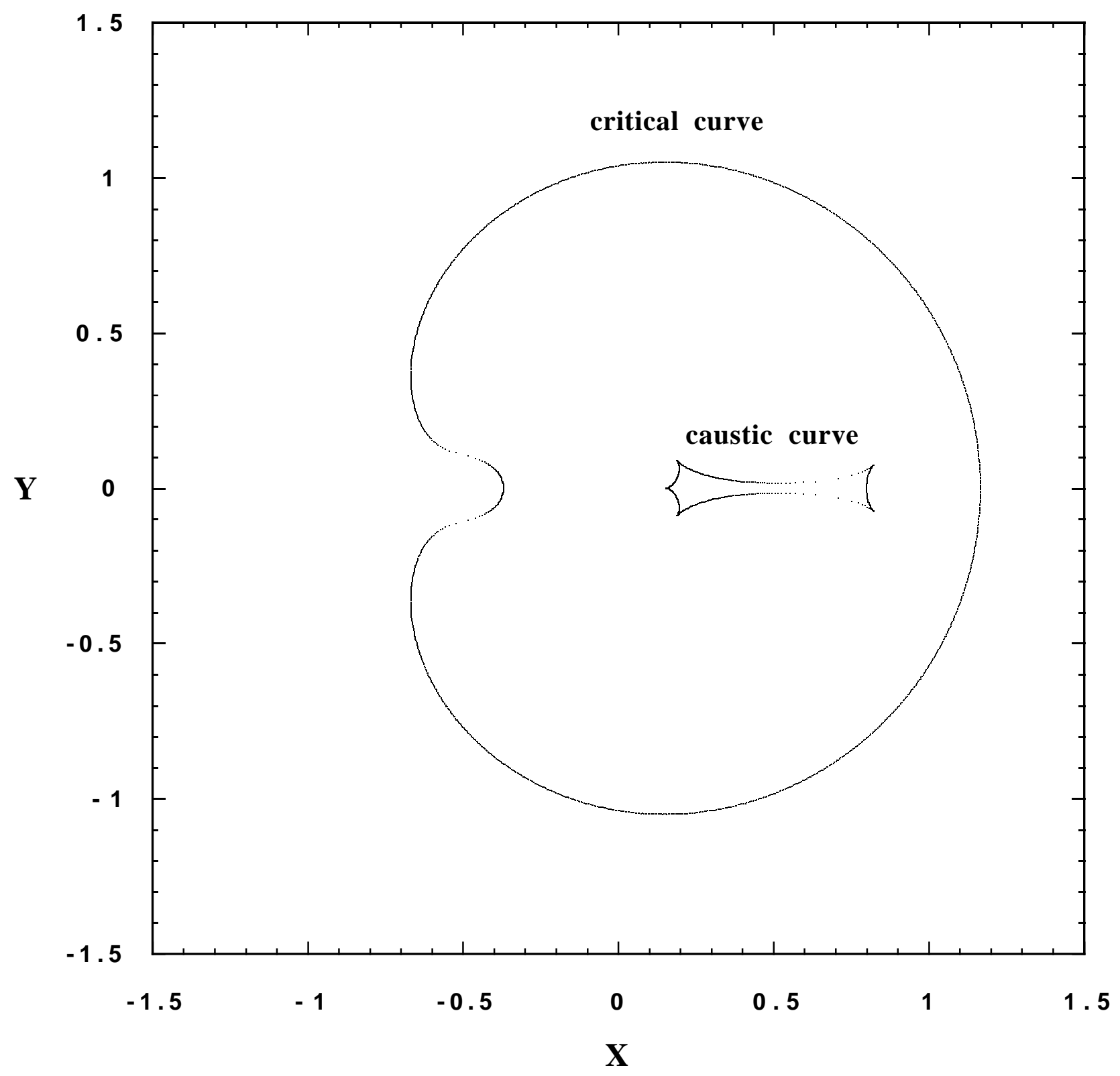


Fig. 4a

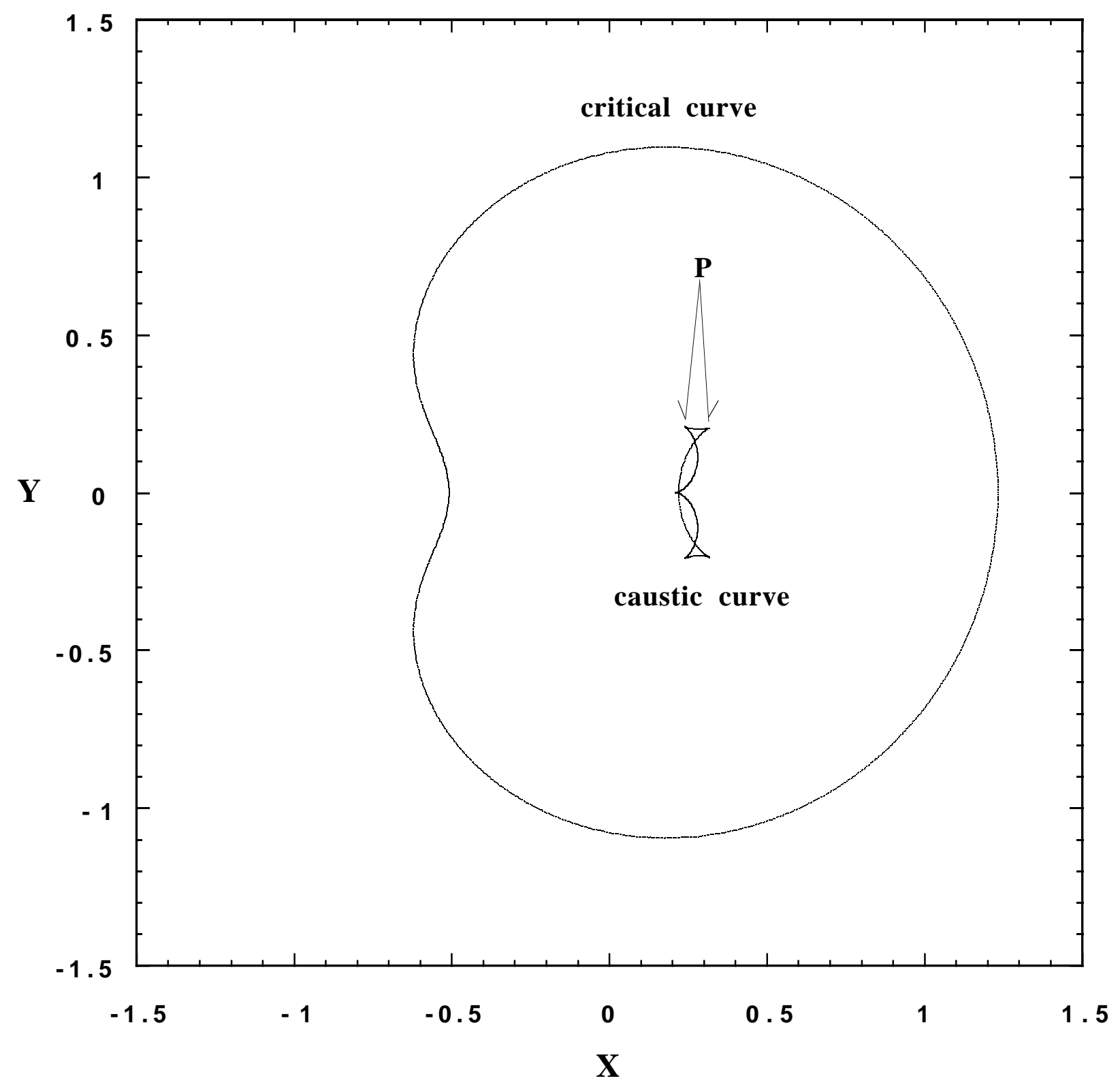


Fig. 4b

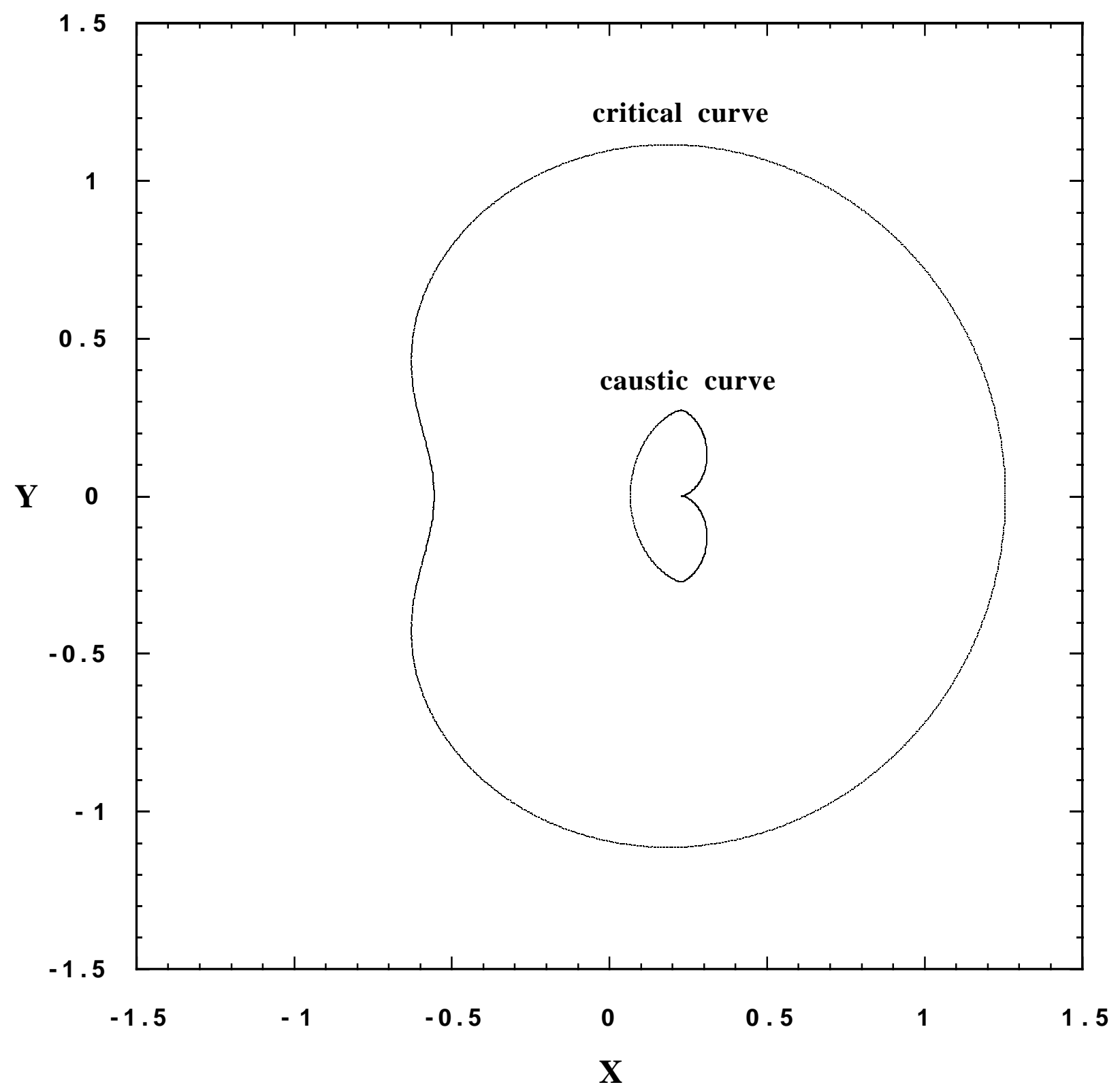


Fig. 4c

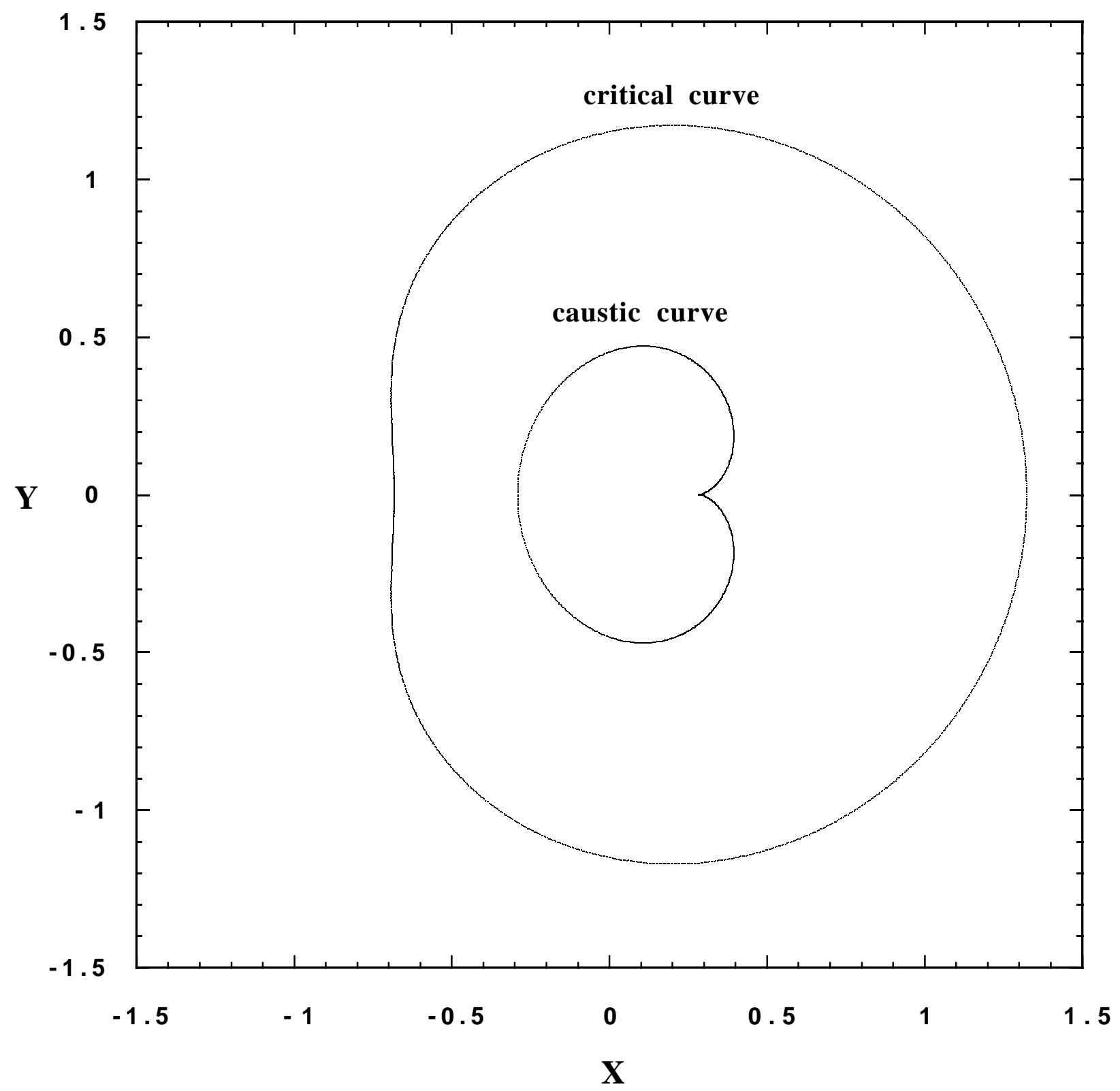


Fig. 5a

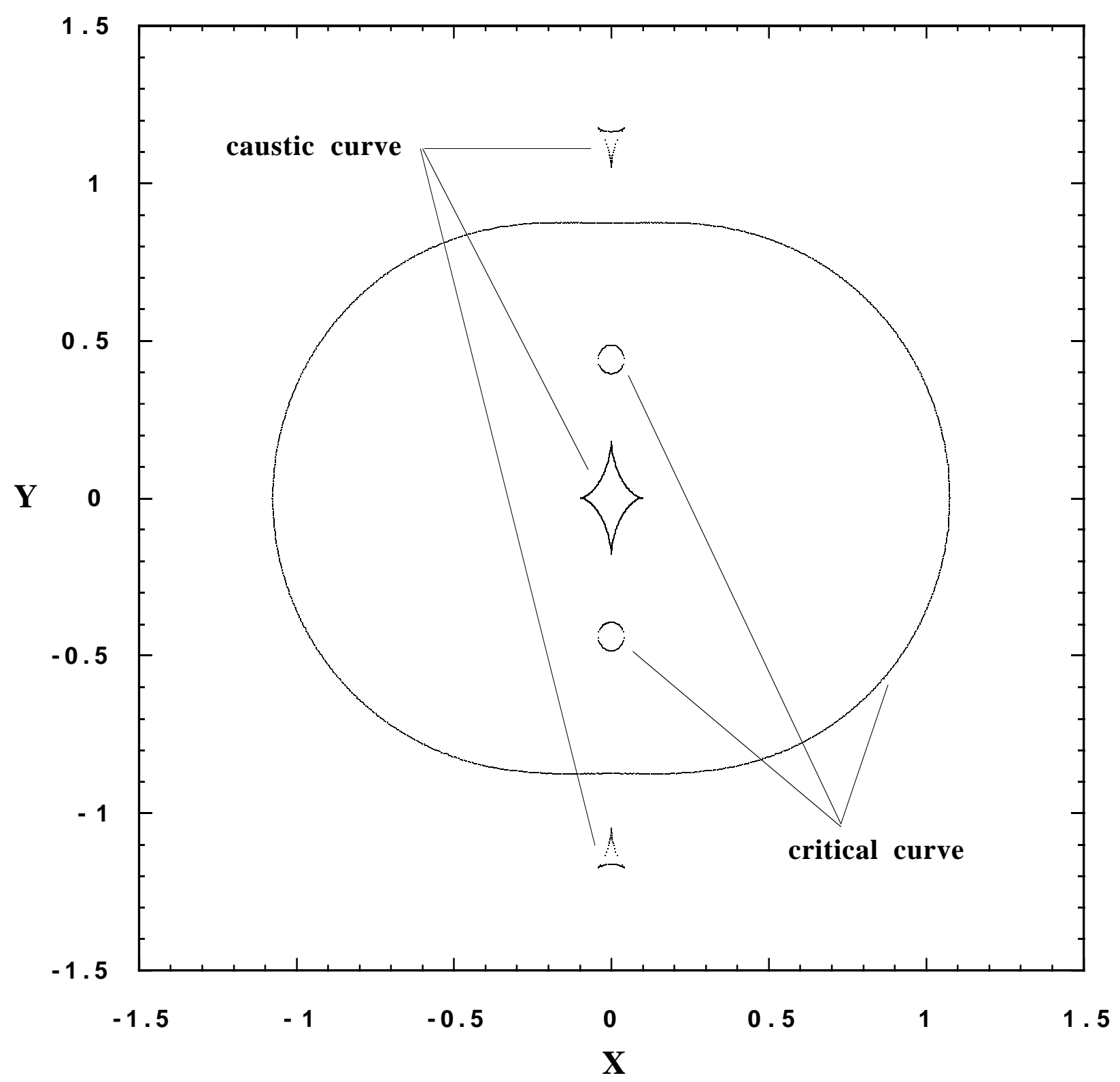


Fig. 5b

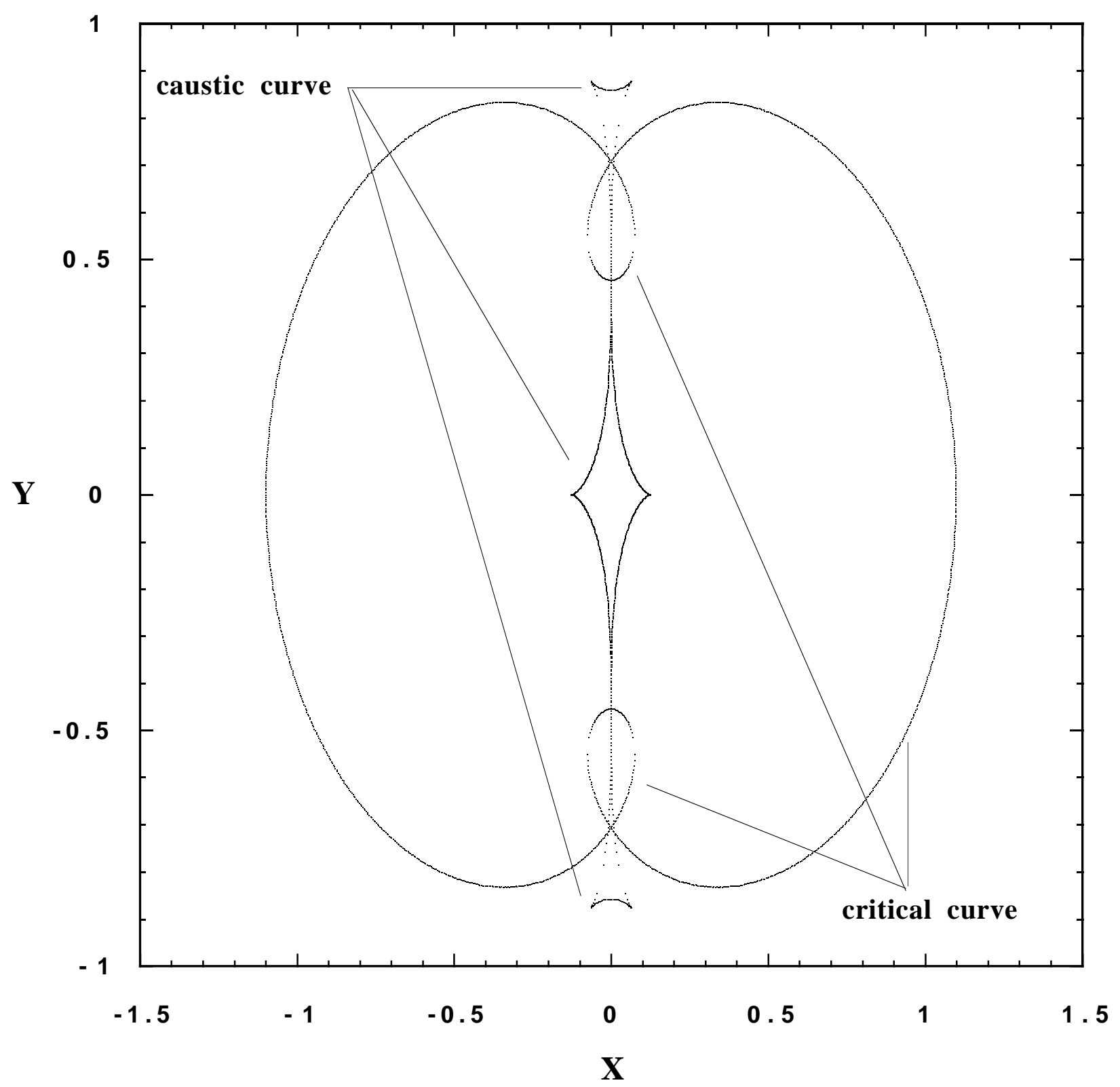


Fig. 5c

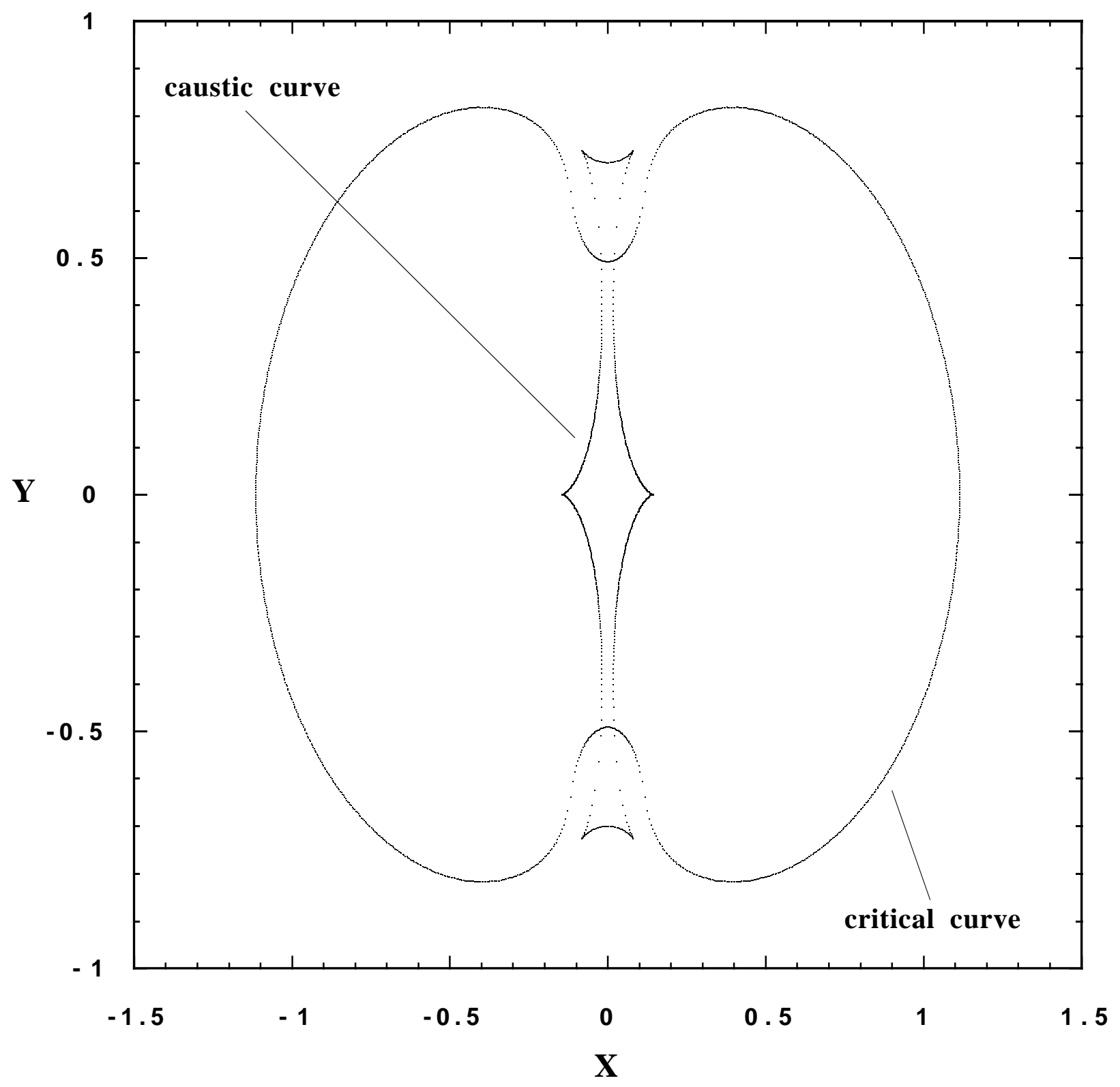


Fig. 6a

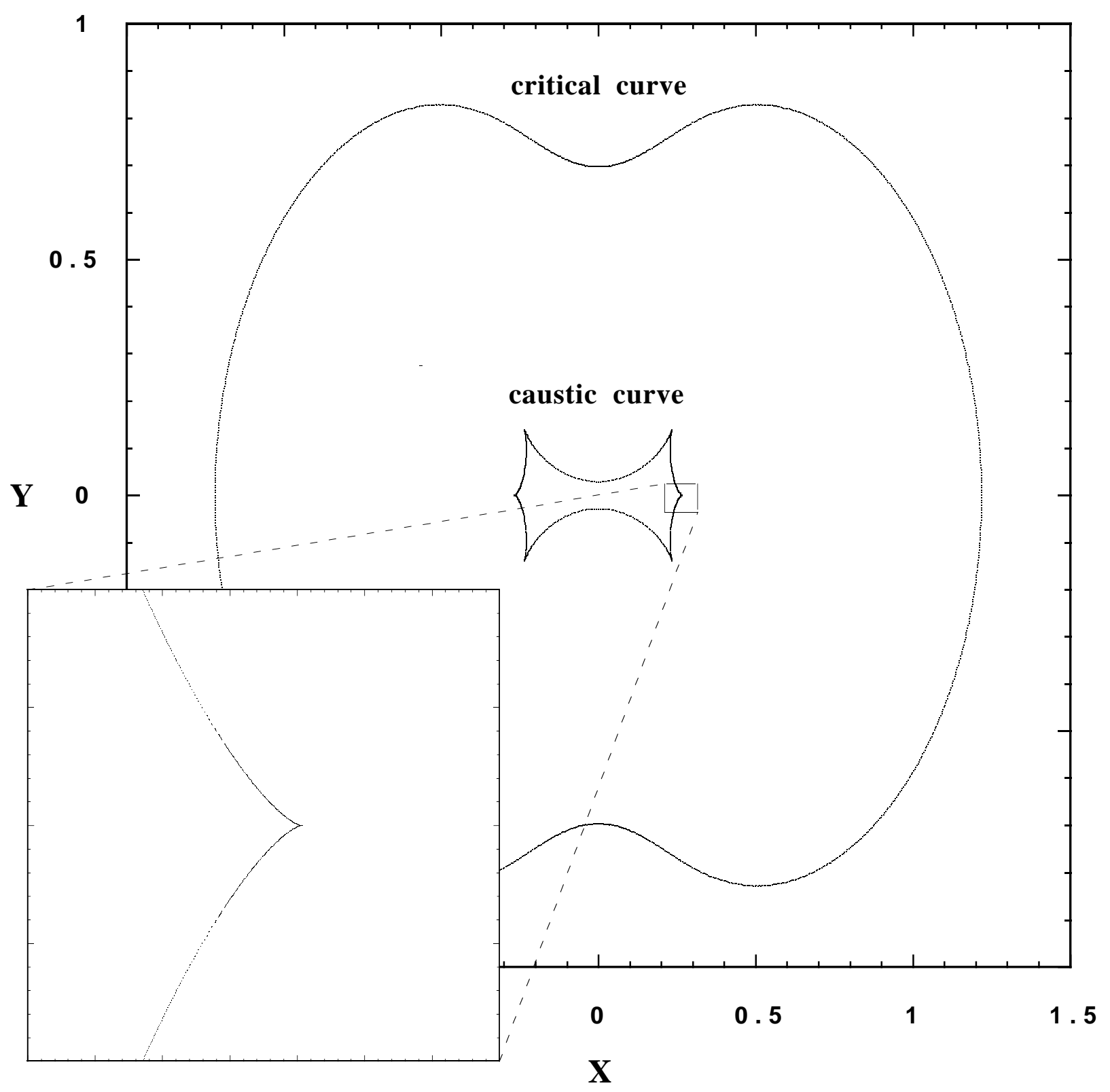


Fig. 6b

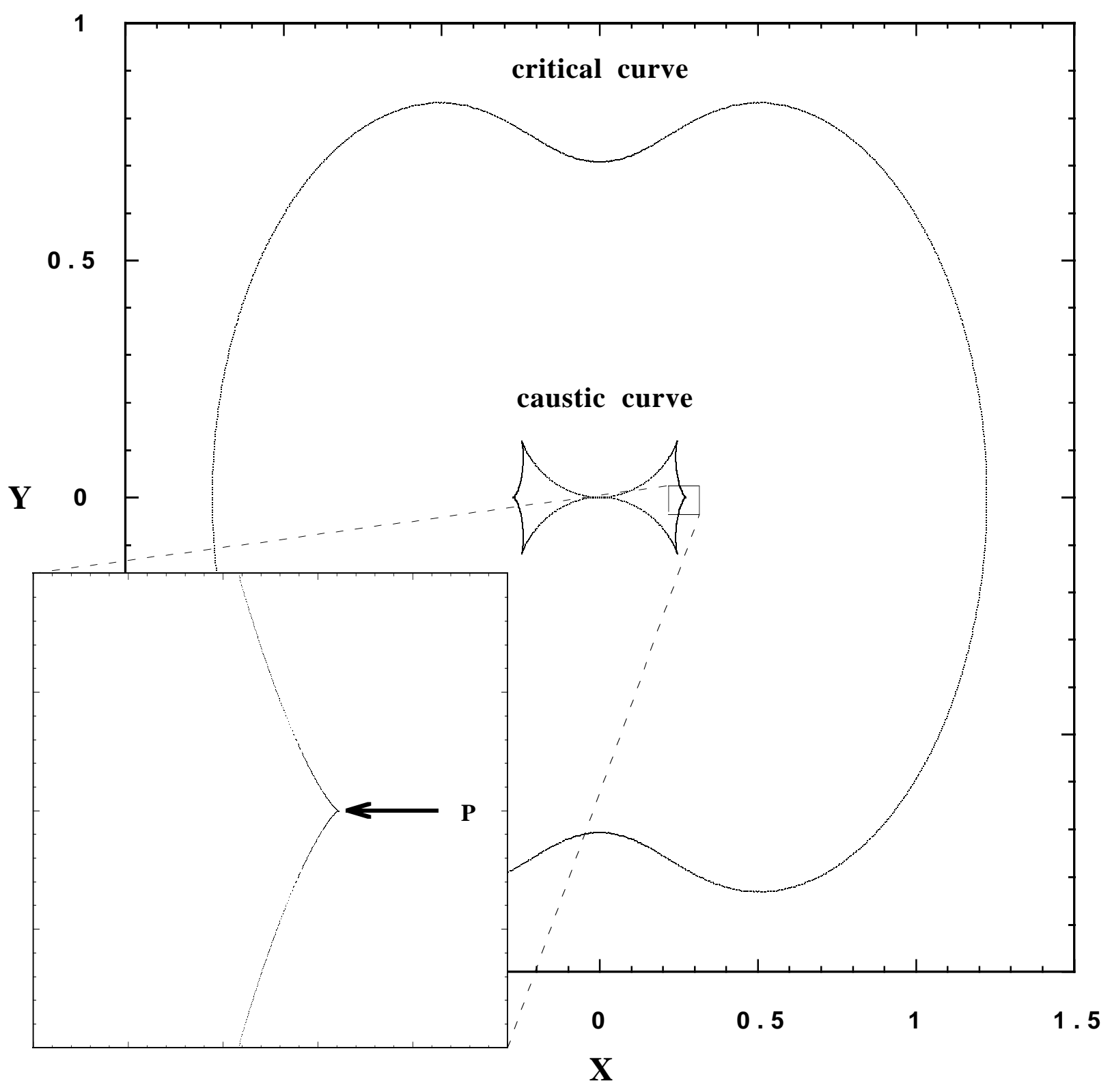


Fig. 6c

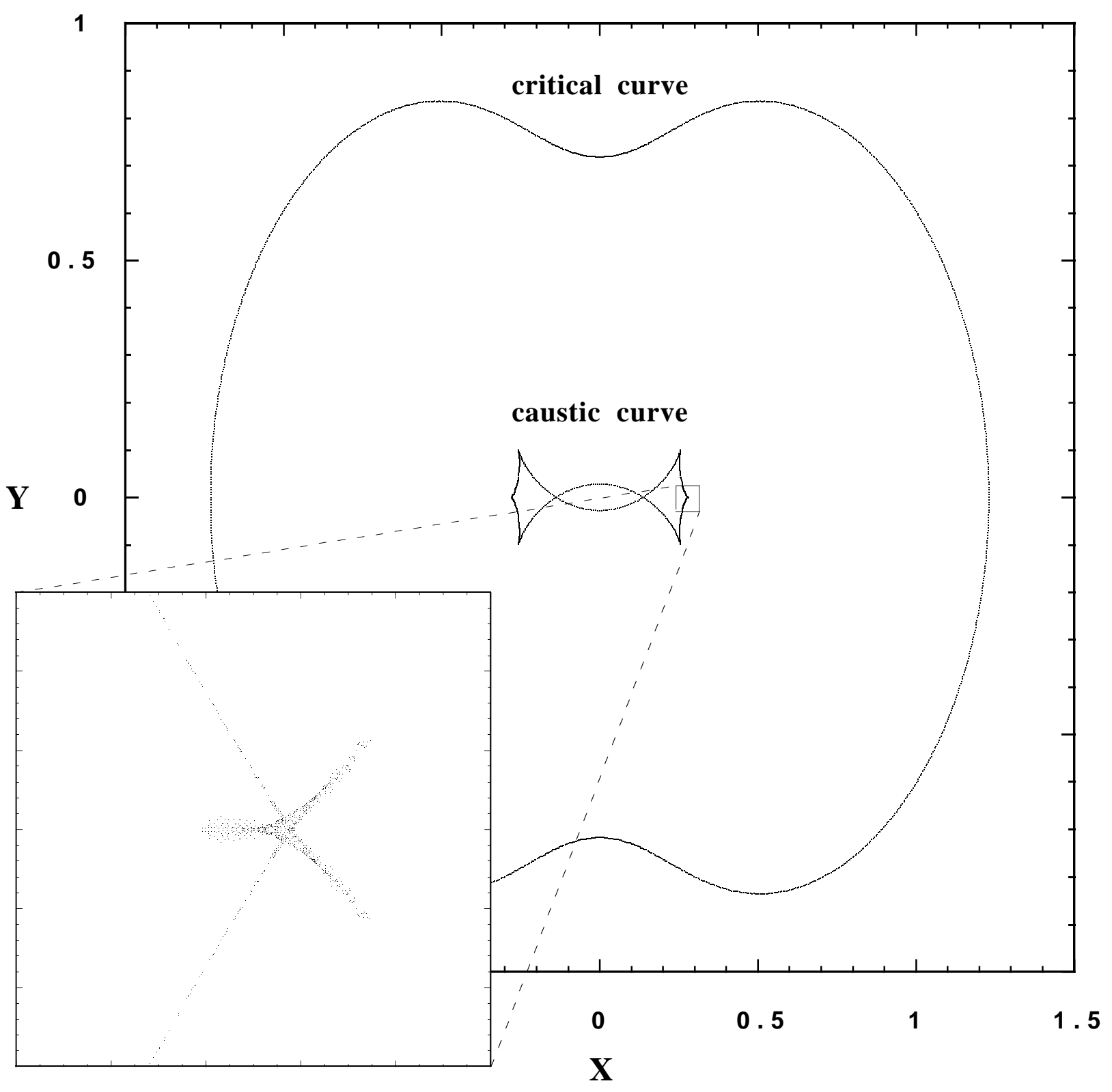


Fig. 7a

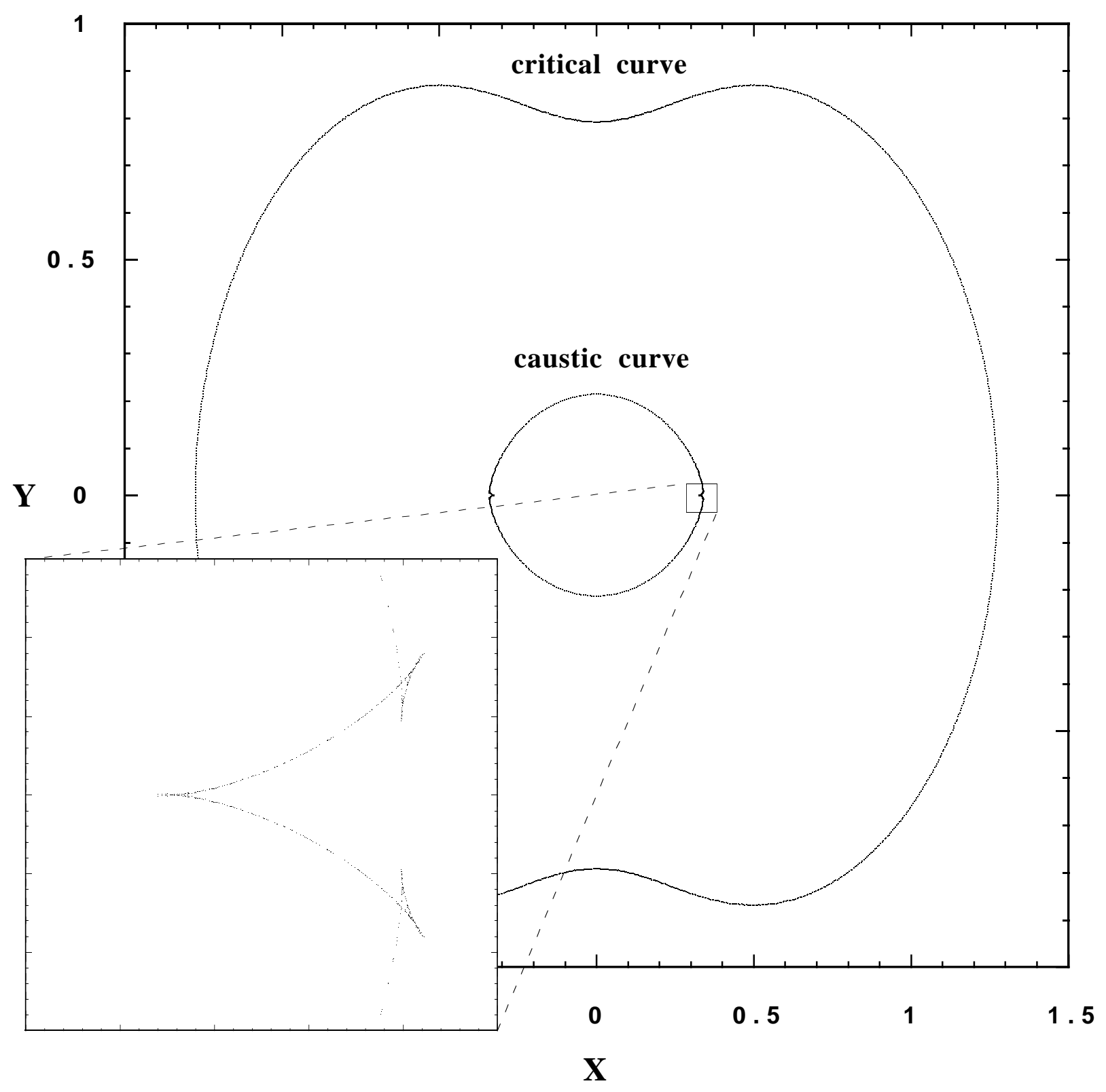


Fig. 7b

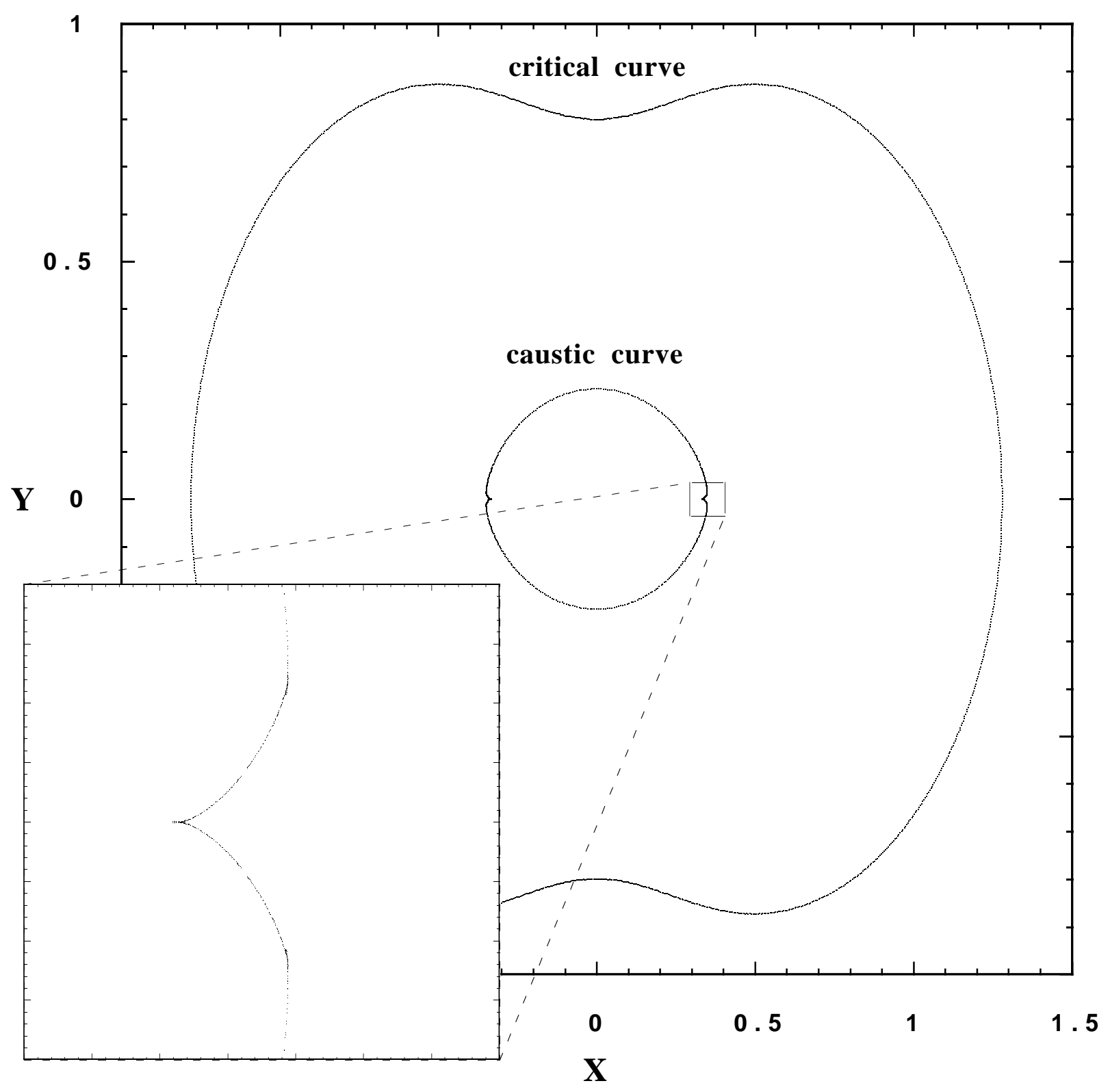


Fig. 7c

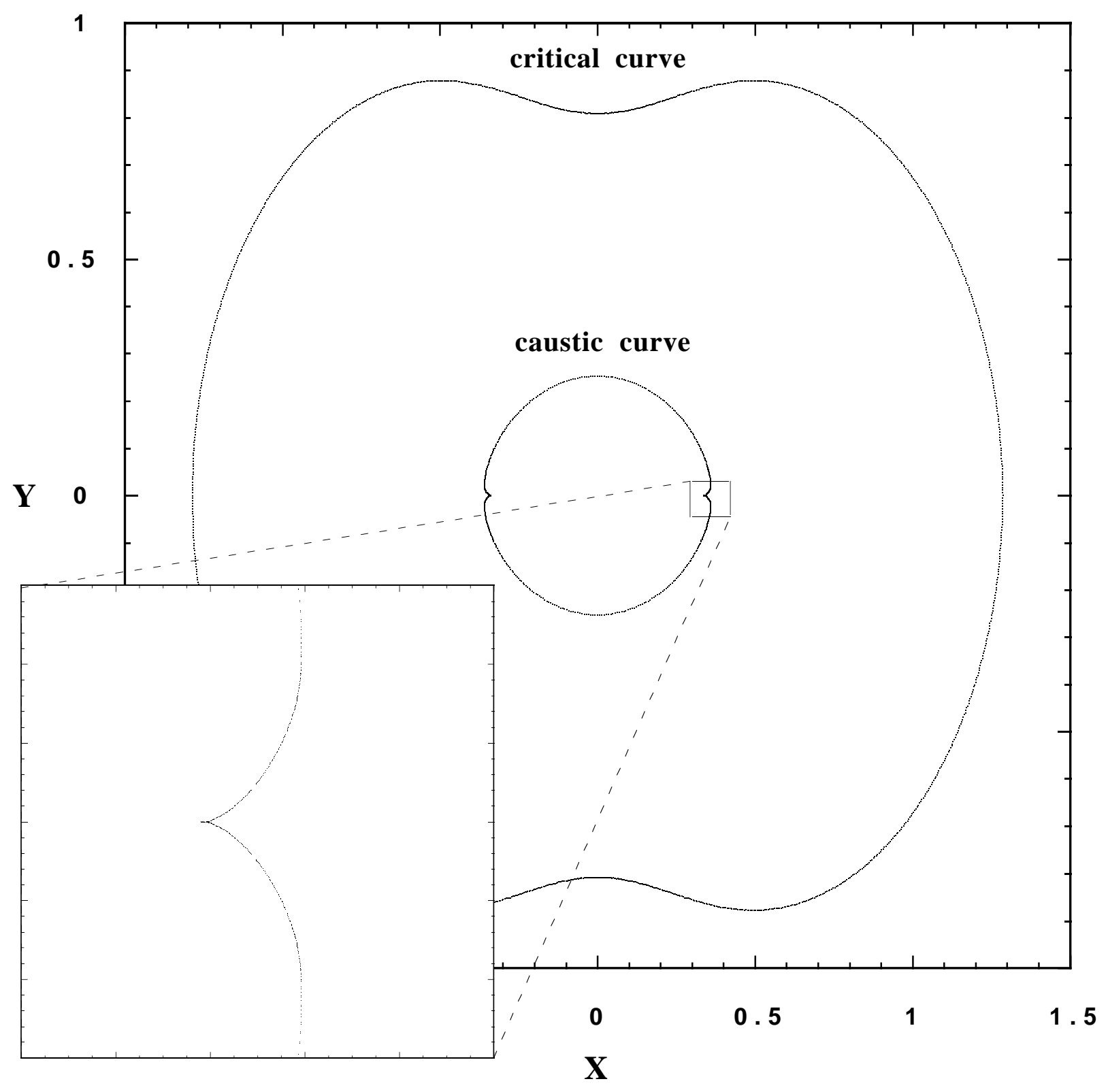


Fig. 8

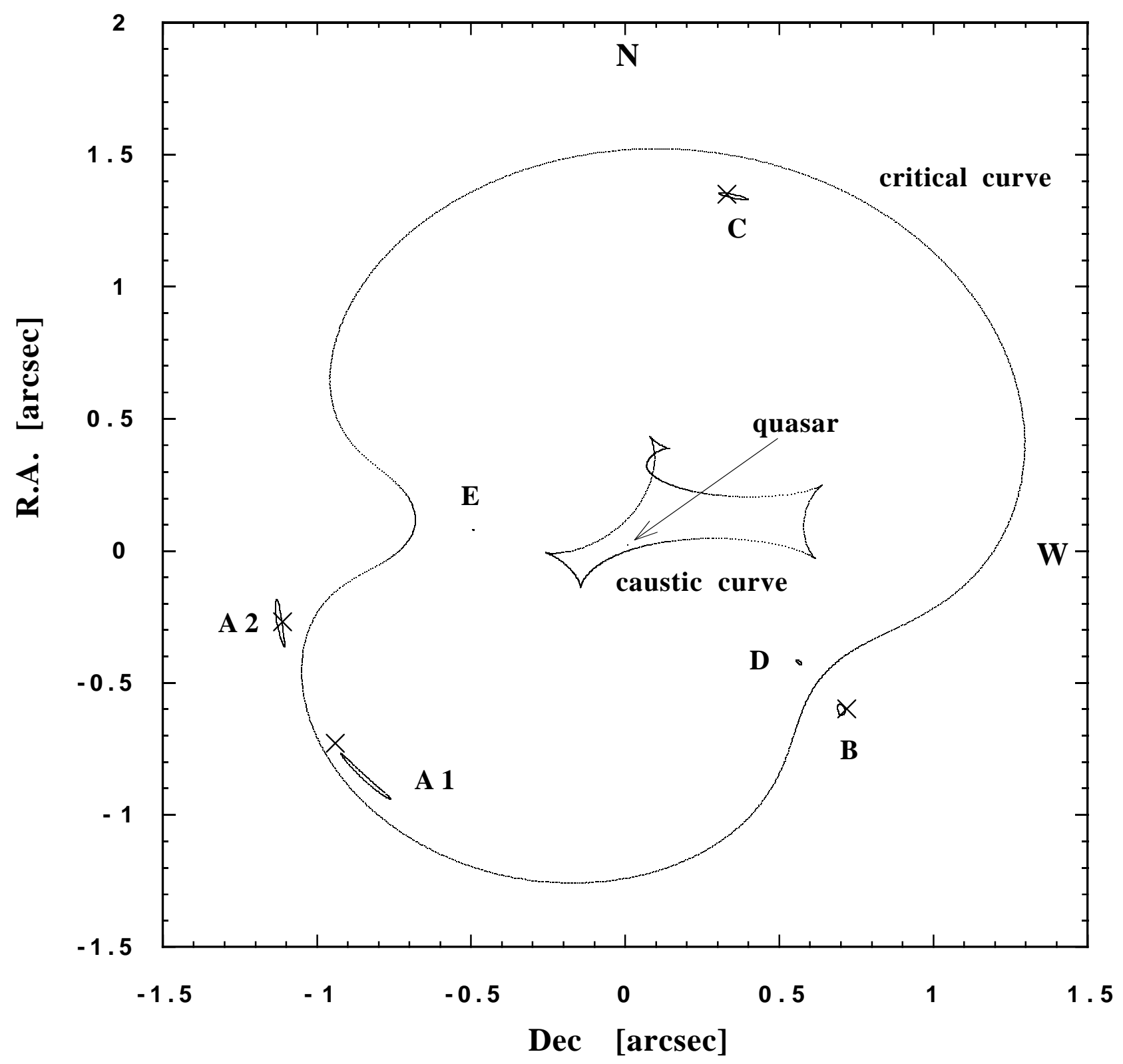

